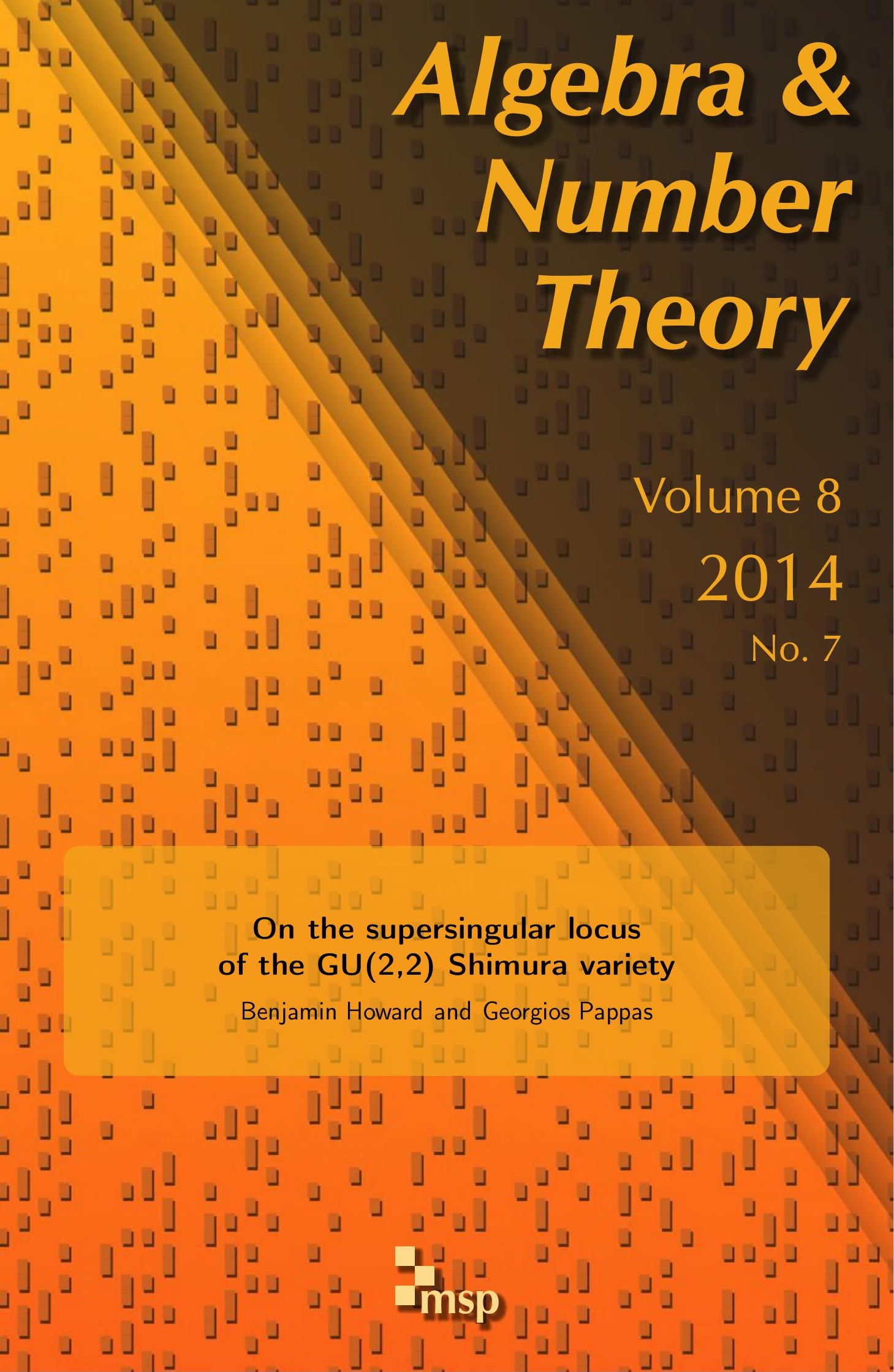




\title{
On the supersingular locus of the $\mathrm{GU}(2,2)$ Shimura variety
}

\author{
Benjamin Howard and Georgios Pappas
}

We describe the supersingular locus of a $\mathrm{GU}(2,2)$ Shimura variety at a prime inert in the corresponding quadratic imaginary field.

\section{Introduction}

This paper contributes to the theory of integral models of Shimura varieties, and, in particular, to the problem of explicitly describing the basic locus in the reduction modulo $p$ of a canonical integral model. In many cases where this integral model is a moduli space of abelian varieties with additional structures, the basic locus coincides with the supersingular locus, i.e., with the subset of the moduli in positive characteristic where the corresponding abelian variety is isogenous to a product of supersingular elliptic curves. The first investigations of a higher-dimensional supersingular locus were for the Siegel moduli space, and are due to Koblitz, Katsura and Oort, and Li and Oort. See the introduction of [Vollaard 2010] for these and other references. More recently, such explicit descriptions for certain unitary and orthogonal Shimura varieties have found applications to Kudla's program relating arithmetic intersection numbers of special cycles on Shimura varieties to Eisenstein series; this motivated further study, as in [Kudla and Rapoport 2009; 1999; 2000; 2011; Vollaard and Wedhorn 2011].

In this paper, we study the supersingular locus of the special fiber of a $\operatorname{GU}(2,2)$ Shimura variety at an odd prime inert in the corresponding imaginary quadratic field. Our methods borrow liberally from [Vollaard 2010] and [Vollaard and Wedhorn 2011], which dealt with the $\mathrm{GU}(n, 1)$ Shimura varieties at inert primes, and from [Rapoport et al. 2014], which considered them at ramified primes. If one attempts to directly imitate the arguments in those papers to study the general $\mathrm{GU}(r, s)$ Shimura variety, the method breaks down at a crucial point. The key new idea for overcoming this obstacle is to exploit the linear algebra underlying a twisted

Pappas is partially supported by NSF grant DMS-1102208; Howard is partially supported by NSF grant DMS-1201480.

MSC2010: primary 11G18; secondary 14G35.

Keywords: Shimura varieties, Deligne-Lusztig varieties. 
version of the exceptional isomorphism $\mathrm{SU}(2,2) \cong \operatorname{Spin}(4,2)$ corresponding to the Dynkin diagram identity $A_{3}=D_{3}$. As such, we do not expect our methods to extend to unitary groups of other signatures (although we do hope that our result will eventually help to predict the shape of the answer in the general case). The problem of understanding the supersingular locus of the $\mathrm{GU}(3,2)$ Shimura variety, for example, remains open.

However, our methods should extend to the family of $\operatorname{GSpin}(n, 2)$ Shimura varieties. Work of Kisin [2010] and Madapusi Pera [2012] (see also [Vasiu 1999]) provides us with a good theory of integral models for these Shimura varieties, and recent work of W. Kim [2013] gives a good theory of Rapoport-Zink spaces as well. An extension of our results in this direction would have applications to Kudla's program, for example by allowing one to generalize the work of Kudla and Rapoport [1999; 2000] from GSpin(2,2) and GSpin $(3,2)$ Shimura varieties to the general $\operatorname{GSpin}(n, 2)$ case. Using the isomorphism between $\operatorname{GSpin}(6,2)$ and the similitude group of a 4-dimensional symplectic module over the Hamiltonian quaternions [Freitag and Hermann 2000], one could also expect to generalize Bültel's results [2012] on the supersingular locus of the moduli space of polarized abelian eightfolds with an action of a definite quaternion algebra. More ambitiously, one could hope to exploit the connection between polarized K3 surfaces and the GSpin(19,2) Shimura variety in order to study the moduli space of supersingular K3 surfaces. Some of these topics will be pursued in subsequent papers.

As this paper was being prepared, Görtz and He were conducting a general study of basic minuscule affine Deligne-Lusztig varieties for equicharacteristic discrete valued fields. The preprint [Göertz and He 2013] provides a list of cases where these affine Deligne-Lusztig varieties can be expressed as a union of usual Deligne-Lusztig varieties, and that list contains an equicharacteristic analogue of the GU(2,2) Rapoport-Zink space considered here. These results of Görtz and He in the equicharacteristic case are analogous to our mixed characteristic results.

1.1. The local result. Our main result concerns the structure of the RapoportZink space parametrizing quasi-isogenies between certain $p$-divisible groups with extra structure. Fix an algebraically closed field $k$ of characteristic $p>2$, let $W$ be the ring of Witt vectors over $k$, and let $E / \mathbb{Q}_{p}$ be an unramified degree-two extension. Consider the family of triples $(G, \iota, \lambda)$, defined over $W$-schemes $S$ on which $p$ is locally nilpotent, consisting of a supersingular $p$-divisible group $G$ with an action $\iota: \mathcal{O}_{E} \rightarrow \operatorname{End}(G)$ and a principal polarization $\lambda: G \rightarrow G^{\vee}$. We require that the action $\iota$ and the polarization $\lambda$ be compatible in the sense of (2-1), and that the action of $\mathcal{O}_{E}$ on $\operatorname{Lie}(G)$ satisfy the signature- $(2,2)$ determinant condition of (2-2). A choice of one such triple $(\boldsymbol{G}, \boldsymbol{\iota}, \boldsymbol{\lambda})$ over $k$ as a basepoint determines the Rapoport-Zink space, $\mathscr{M}$, parametrizing quadruples $(G, \iota, \lambda, \varrho)$ in 
which $\varrho: G \times_{S} S_{0} \rightarrow \boldsymbol{G} \times_{k} S_{0}$ is an $\mathcal{O}_{E}$-linear quasi-isogeny under which $\lambda$ pulls back to a $\mathbb{Q}_{p}^{\times}$-multiple $c(\varrho) \lambda$. Here, $S_{0}=S \times_{W} k$. The Rapoport-Zink space $\mathscr{M}$ is a formal scheme over $W$, and admits a decomposition into open and closed formal subschemes $\mathscr{M}=\biguplus_{\ell \in \mathbb{Z}} \mathscr{M}^{(\ell)}$, where $\mathscr{M}^{(\ell)}$ is the locus where $\operatorname{ord}_{p}(c(\varrho))=\ell$. Here and elsewhere, we use the symbol $\biguplus$ to denote disjoint union. The group $p^{\mathbb{Z}}$ acts on $\mathscr{M}$, where the action of $p$ sends $(G, \iota, \lambda, \varrho) \mapsto(G, \iota, \lambda, p \varrho)$. This action has $\mathscr{M}^{(0)} \uplus \mathscr{M}^{(1)}$ as a fundamental domain. In fact, the action of $p^{\mathbb{Z}}$ extends to a larger group $J$ which acts transitively on the set $\left\{\mathscr{M}^{(\ell)}: \ell \in \mathbb{Z}\right\}$. Define

$$
\mathscr{N}=p^{\mathbb{Z}} \backslash \mathscr{M},
$$

and let $\mathscr{N}^{+}$and $\mathscr{N}^{-}$be the images of $\mathscr{M}^{(0)}$ and $\mathscr{M}^{(1)}$, respectively, under the quotient map $\mathscr{M} \rightarrow \mathscr{N}$.

In Section 2, we construct a 6-dimensional $\mathbb{Q}_{p}$-vector space

$$
\boldsymbol{L}_{\mathbb{Q}}^{\Phi} \subset \operatorname{End}(\boldsymbol{G})_{\mathbb{Q}}
$$

of special quasi-endomorphisms of $\boldsymbol{G}$ as the $\Phi$-fixed vectors in a slope-0 isocrystal $\left(\boldsymbol{L}_{\mathbb{Q}}, \Phi\right)$. The vector space $\boldsymbol{L}_{\mathbb{Q}}^{\Phi}$ is endowed with a $\mathbb{Q}_{p}$-valued quadratic form $Q(x)=$ $x \circ x$, and we define a vertex lattice in $\boldsymbol{L}_{\mathbb{Q}}^{\Phi}$ to be a $\mathbb{Z}_{p}$-lattice $\Lambda \subset \boldsymbol{L}_{\mathbb{Q}}^{\Phi}$ such that

$$
p \Lambda \subset \Lambda^{\vee} \subset \Lambda \text {. }
$$

The type $t_{\Lambda} \in\{2,4,6\}$ of $\Lambda$ is the dimension of $\Lambda / \Lambda^{\vee}$. To each point $(G, \iota, \lambda, \varrho)$ of $\mathscr{N}$, the quasi-isogeny $\varrho$ allows us to view $\Lambda$ as a lattice of quasi-endomorphisms of $G$. Let $\widetilde{N}_{\Lambda} \subset \mathscr{N}$ be the locus of points where $\Lambda \subset \operatorname{End}(G)$ (i.e., the locus where these quasi-endomorphisms are integral). It is a closed formal subscheme of $\mathscr{N}$, whose underlying reduced $k$-scheme we denote by $\mathscr{N}_{\Lambda}$. We show that the underlying reduced subscheme $\mathscr{N}_{\text {red }}$ of $\mathscr{N}$ is covered by these closed subschemes:

$$
\mathscr{N}_{\text {red }}=\bigcup_{\Lambda} \mathscr{N}_{\Lambda}
$$

and that

$$
\mathscr{N}_{\Lambda_{1}} \cap \mathscr{N}_{\Lambda_{2}}= \begin{cases}\mathscr{N}_{\Lambda_{1} \cap \Lambda_{2}} & \text { if } \Lambda_{1} \cap \Lambda_{2} \text { is a vertex lattice, } \\ \varnothing & \text { otherwise, }\end{cases}
$$

where the left-hand side is understood to mean the reduced subscheme underlying the scheme-theoretic intersection (we suspect that the scheme-theoretic intersection is already reduced, but are unable to provide a proof).

Section 3 is devoted to understanding the structure of $\mathscr{N}_{\Lambda}^{ \pm}=\mathscr{N}_{\Lambda} \cap \mathscr{N}^{ \pm}$. Setting $d_{\Lambda}=t_{\Lambda} / 2$, we prove that $\mathscr{N}_{\Lambda}^{ \pm}$is a projective, smooth, and irreducible $k$-scheme of dimension $d_{\Lambda}-1$. In fact:

(1) If $d_{\Lambda}=1$, then $\mathscr{N}_{\Lambda}^{ \pm}$is a single point. 
(2) If $d_{\Lambda}=2$, then $\mathscr{N}_{\Lambda}^{ \pm}$is isomorphic to $\mathbb{P}^{1}$.

(3) If $d_{\Lambda}=3$, then $\mathscr{N}_{\Lambda}^{ \pm}$is isomorphic to the Fermat hypersurface

$$
x_{0}^{p+1}+x_{1}^{p+1}+x_{2}^{p+1}+x_{3}^{p+1}=0 .
$$

The irreducible components of $\mathscr{N}^{ \pm}$are precisely the closed subschemes $\mathscr{N}_{\Lambda}^{ \pm}$ indexed by the type- 6 vertex lattices. From this we deduce the following theorem: Theorem A. The underlying reduced scheme $\mathscr{M}_{\text {red }}^{(\ell)}$ of $\mathscr{M}^{(\ell)}$ is connected. Every irreducible component of $\mathscr{M}_{\text {red }}^{(\ell)}$ is a smooth $k$-scheme of dimension 2, isomorphic to the Fermat hypersurface

$$
x_{0}^{p+1}+x_{1}^{p+1}+x_{2}^{p+1}+x_{3}^{p+1}=0 .
$$

If two irreducible components intersect nontrivially, the reduced scheme underlying their scheme-theoretic intersection is either a point or a projective line.

See Sections 3.5 and 3.6 for a more detailed description of $\mathscr{M}_{\text {red }}$.

1.2. The global result. In Section 4 , we consider the global situation. Let $E$ be a quadratic imaginary field, and let $p>2$ be inert in $E$. Let $\mathcal{O} \subset E$ be the integral closure of $\mathbb{Z}_{(p)}$, and let $V$ be a free $\mathcal{O}$-module of rank 4 endowed with a perfect $\mathcal{O}$-valued Hermitian form of signature $(2,2)$. Let $G=\mathrm{GU}(V)$ be the group of unitary similitudes of $V$, a reductive group over $\mathbb{Z}_{(p)}$. Fix a compact open subgroup $U^{p} \subset G\left(\mathbb{A}_{f}^{p}\right)$, which we assume is sufficiently small, and define $U_{p}=G\left(\mathbb{Z}_{p}\right)$ and $U=U_{p} U^{p} \subset G\left(\mathbb{A}_{f}\right)$.

Using this data we define a scheme $M_{U}$, smooth of relative dimension 4 over $\mathbb{Z}_{(p)}$, as a moduli space of abelian fourfolds, up to prime-to- $p$-isogeny, with additional structure, in such a way that the complex fiber of $M_{U}$ is the Shimura variety

$$
M_{U}(\mathbb{C})=G(\mathbb{Q}) \backslash\left(\mathcal{D} \times G\left(\mathbb{A}_{f}\right) / U\right) .
$$

Here, $\mathcal{D}$ is the Grassmannian of negative-definite planes in $V \otimes_{\mathcal{O}} \mathbb{C}$.

Let $k$ be an algebraically closed field of characteristic $p$, and denote by $M_{U}^{\text {ss }}$ the reduced supersingular locus of the geometric special fiber $M_{U} \times_{\mathbb{Z}_{(p)}} k$. The uniformization theorem of Rapoport and Zink expresses $M_{U}^{\text {ss }}$ as a disjoint union of quotients of the scheme $\mathscr{M}_{\text {red }}$ described above. As a consequence we obtain the following result:

Theorem B. The $k$-scheme $M_{U}^{\text {ss }}$ has pure dimension 2. For $U^{p}$ sufficiently small, all irreducible components of $M_{U}^{\mathrm{ss}}$ are isomorphic to the Fermat hypersurface

$$
x_{0}^{p+1}+x_{1}^{p+1}+x_{2}^{p+1}+x_{3}^{p+1}=0 .
$$

If two irreducible components intersect nontrivially, the reduced scheme underlying their scheme-theoretic intersection is either a point or a projective line. 
1.3. Notation. We use the following notation throughout Sections 2 and 3. Fix an odd prime $p$ and an unramified quadratic extension $E$ of the field of $p$-adic numbers $\mathbb{Q}_{p}$. The nontrivial Galois automorphism of $E$ is denoted by $\alpha \mapsto \bar{\alpha}$. Let $k$ be an algebraically closed field of characteristic $p$. Its ring of Witt vectors $W=W(k)$ is a complete discrete valuation ring with residue field $k=W / p W$ and fraction field $W_{\mathbb{Q}}$. Label the two embeddings of $\mathcal{O}_{E}$ into $W$ as

$$
\psi_{0}: \mathcal{O}_{E} \rightarrow W, \quad \psi_{1}: \mathcal{O}_{E} \rightarrow W,
$$

and denote by $\sigma$ both the absolute Frobenius $x \mapsto x^{p}$ on $k$ and its unique lift to a ring automorphism of $W$. Denote by $\epsilon_{0}, \epsilon_{1} \in \mathcal{O}_{E} \otimes W$ the orthogonal idempotents characterized by

$$
\epsilon_{i} M=\left\{x \in M:(\alpha \otimes 1) \cdot x=\left(1 \otimes \psi_{i}(\alpha)\right) \cdot x \text { for all } \alpha \in \mathcal{O}_{E}\right\}
$$

for any $\mathcal{O}_{E} \otimes W$-module $M$. For any $\mathbb{Z}$-module $M$, we abbreviate $M_{\mathbb{Q}}=M \otimes_{\mathbb{Z}} \mathbb{Q}$. In particular, $M_{\mathbb{Q}}=M \otimes_{W} W[1 / p]$ for any $W$-module $M$.

\section{Moduli spaces and lattices}

In this section we recall the Rapoport-Zink space of a GU(2,2) Shimura variety, and define a stratification of the underlying reduced scheme.

2.1. The Rapoport-Zink space. Let $\mathrm{Nilp}_{W}$ be the category of $W$-schemes on which $p$ is locally nilpotent. We wish to parametrize triples $(G, \iota, \lambda)$ over objects $S$ of Nilp $_{W}$ in which

- $G$ is a supersingular $p$-divisible group of dimension 4,

- $\iota: \mathcal{O}_{E} \rightarrow \operatorname{End}(G)$ is an action of $\mathcal{O}_{E}$ on $G$,

- $\lambda: G \rightarrow G^{\vee}$ is a principal polarization.

We further require that every $\alpha \in \mathcal{O}_{E}$ satisfy both the $\mathcal{O}_{E}$-linearity condition

$$
\lambda \circ \iota(\bar{\alpha})=\iota(\alpha)^{\vee} \circ \lambda
$$

and the signature- $(2,2)$ condition

$$
\operatorname{det}(T-\iota(\alpha) ; \operatorname{Lie}(G))=\left(T-\psi_{0}(\alpha)\right)^{2}\left(T-\psi_{1}(\alpha)\right)^{2}
$$

as sections of $\mathcal{O}_{S}[T]$. The signature- $(2,2)$ condition is equivalent to each of the $\mathcal{O}_{S}$-module direct summands in $\operatorname{Lie}(G)=\epsilon_{0} \operatorname{Lie}(G) \oplus \epsilon_{1} \operatorname{Lie}(G)$ being locally free of rank 2.

Fix one such triple $(\boldsymbol{G}, \boldsymbol{\iota}, \lambda)$ over $k$ as a base point, and let $\mathscr{M}$ be the functor on Nilp $_{W}$ sending $S$ to the set of isomorphism classes of quadruples $(G, \lambda, i, \varrho)$ over $S$, where $(G, \iota, \lambda)$ is as above and $\varrho: G_{/ S_{0}} \rightarrow \boldsymbol{G}_{/ S_{0}}$ is an $\mathcal{O}_{E}$-linear quasi-isogeny such that $\varrho^{*} \lambda=c(\varrho) \lambda$ for some $c(\varrho) \in \mathbb{Q}_{p}^{\times}$. Here, $S_{0}$ is the $k$-scheme $S \otimes_{W} k$. 
The functor $\mathscr{M}$ is represented by a formal scheme locally of finite type over $\operatorname{Spf}(W)$ by [Rapoport and Zink 1996]. There is a decomposition $\mathscr{M}=\biguplus_{\ell \in \mathbb{Z}} \mathscr{M}^{(\ell)}$ into open and closed formal subschemes, where $\mathscr{M}^{(\ell)}$ is the locus of points where $\operatorname{ord}_{p}(c(\varrho))=\ell$.

Let $J \subset \operatorname{End}(\boldsymbol{G})_{\mathbb{Q}}^{\times}$denote the subgroup of $E$-linear elements such that $g^{*} \boldsymbol{\lambda}=$ $\nu(g) \lambda$ for some $v(g) \in \mathbb{Q}_{p}^{\times}$. The group $J$ acts on $\mathscr{M}$ in an obvious way:

$$
g \cdot(G, \iota, \lambda, \varrho)=(G, \iota, \lambda, g \circ \varrho) .
$$

As usual, the group $J$ is the $\mathbb{Q}_{p}$-points of a reductive group over $\mathbb{Q}_{p}$. In fact, by [Vollaard 2010, Remark 1.16], this reductive group is the group of unitary similitudes of the split Hermitian space of dimension 4 over $E$. In particular, the derived subgroup $J^{\text {der }}$ is isomorphic to the special unitary group, and the similitude character $v: J \rightarrow \mathbb{Q}_{p}^{\times}$is surjective. Note that the action of any $g \in J$ with $\operatorname{ord}_{p}(v(g))=1$ defines an isomorphism $\mathscr{M}^{(\ell)} \cong \mathscr{M}^{(\ell+1)}$.

As a special case of this action, the group $p^{\mathbb{Z}}$ acts on $\mathscr{M}$ by

$$
p \cdot(G, \iota, \lambda, \varrho)=(G, \iota, \lambda, p \varrho),
$$

and the quotient $\mathscr{N}=p^{\mathbb{Z}} \backslash \mathscr{M}$ has $\mathscr{M}^{(0)} \uplus \mathscr{M}^{(1)}$ as a fundamental domain. Let $\mathscr{N}^{+} \cong \mathscr{M}^{(0)}$ and $\mathscr{N}^{-} \cong \mathscr{M}^{(1)}$ be the open and closed formal subschemes of $\mathscr{N}$ on which $\operatorname{ord}_{p}(c(\varrho))$ is even and odd, respectively. By the previous paragraph there is an isomorphism $\mathscr{N}^{+} \cong \mathscr{N}^{-}$, and we will see later in Theorem 3.12 that $\mathscr{N}^{+}$ and $\mathscr{N}^{-}$are precisely the connected components of $\mathscr{N}$.

2.2. Special endomorphisms. In this subsection we will define a $\mathbb{Q}_{p}$-subspace

$$
\boldsymbol{L}_{\mathbb{Q}}^{\Phi} \subset \operatorname{End}(\boldsymbol{G})_{\mathbb{Q}}
$$

of special quasi-endomorphisms of $\boldsymbol{G}$ in such a way that $x \mapsto x \circ x$ defines a $\mathbb{Q}_{p}$-valued quadratic form on $\boldsymbol{L}_{\mathbb{Q}}^{\Phi}$. The subspace $\boldsymbol{L}_{\mathbb{Q}}^{\Phi}$ is not quite canonical; it will depend on the auxiliary choice of a certain tensor $\omega$ in the top exterior power of the Dieudonné module of $\boldsymbol{G}$.

Denote by $\boldsymbol{D}$ the covariant Dieudonné module of $\boldsymbol{G}$, with its induced action of $\mathcal{O}_{E}$ and induced alternating form $\lambda: \bigwedge_{W}^{2} \boldsymbol{D} \rightarrow W$ satisfying $\lambda(F x, y)=$ $\lambda(x, V y)^{\sigma}$. Under the covariant conventions, $\operatorname{Lie}(\boldsymbol{G}) \cong \boldsymbol{D} / V \boldsymbol{D}$ as $k$-vector spaces with $\mathcal{O}_{E}$-actions. Abbreviate $\wedge_{E}^{\ell} \boldsymbol{D}=\bigwedge_{\mathcal{O}_{E} \otimes W}^{\ell} \boldsymbol{D}$. Once we fix a $\delta \in \mathcal{O}_{E}^{\times}$satisfying $\delta^{\sigma}=-\delta$, there is a unique Hermitian form

$$
\langle\cdot, \cdot\rangle: \boldsymbol{D} \times \boldsymbol{D} \rightarrow \mathcal{O}_{E} \otimes W
$$

satisfying

$$
\lambda(x, y)=\operatorname{Tr}_{E / \mathbb{Q}_{p}} \delta^{-1}\langle x, y\rangle,
$$


which in turn induces a Hermitian form on every exterior power $\wedge_{E}^{\ell} \boldsymbol{D}$ by

$$
\left\langle x_{1} \wedge \cdots \wedge x_{\ell}, y_{1} \wedge \cdots \wedge y_{\ell}\right\rangle=\sum_{\pi \in S_{\ell}} \operatorname{sgn}(\pi) \prod_{i=1}^{\ell}\left\langle x_{i}, y_{\pi(i)}\right\rangle .
$$

This Hermitian form identifies each lattice $\bigwedge_{E}^{\ell} \boldsymbol{D}$ with its dual lattice in $\left(\bigwedge_{E}^{\ell} \boldsymbol{D}\right)_{\mathbb{Q}}$. In order to make explicit calculations, we now put coordinates on $\boldsymbol{D}_{\mathbb{Q}}$.

Lemma 2.1. There are $W_{\mathbb{Q}}$-bases

such that

$$
\begin{gathered}
e_{1}, e_{2}, e_{3}, e_{4} \in \epsilon_{0} \boldsymbol{D}_{\mathbb{Q}}, \\
f_{1}, f_{2}, f_{3}, f_{4} \in \epsilon_{1} \boldsymbol{D}_{\mathbb{Q}},
\end{gathered}
$$

$$
\left\langle e_{i}, f_{j}\right\rangle= \begin{cases}\epsilon_{0} & \text { if } i=j, \\ 0 & \text { otherwise }\end{cases}
$$

and the $\sigma$-semilinear operator $F$ satisfies

$$
\begin{array}{llll}
F e_{1}=f_{1}, & F e_{2}=f_{2}, & F e_{3}=p f_{3}, & F e_{4}=p f_{4}, \\
F f_{1}=p e_{1}, & F f_{2}=p e_{2}, & F f_{3}=e_{3}, & F f_{4}=e_{4} .
\end{array}
$$

Proof. Denote by $\boldsymbol{D}_{\mathbb{Q}}^{\prime}$ the isocrystal with $W_{\mathbb{Q}}$-basis $\left\{e_{1}, \ldots, e_{4}, f_{1}, \ldots, f_{4}\right\}$ and by $F$ the operator defined by the above relations. Endow $\boldsymbol{D}_{\mathbb{Q}}^{\prime}$ with the $E$-action $\iota^{\prime}(\alpha) e_{i}=\psi_{0}(\alpha) e_{i}$ and $\iota^{\prime}(\alpha) f_{i}=\psi_{1}(\alpha) f_{i}$ and the unique Hermitian form satisfying (2-4). This Hermitian form determines a polarization $\lambda^{\prime}(x, y)=\operatorname{Tr}_{E / \mathbb{Q}_{p}} \delta^{-1}\langle x, y\rangle$. As $\boldsymbol{D}_{\mathbb{Q}}^{\prime}$ is isoclinic of slope $1 / 2$, there is an isomorphism of isocrystals

$$
\varrho: \boldsymbol{D}_{\mathbb{Q}} \cong \boldsymbol{D}_{\mathbb{Q}}^{\prime} .
$$

Any two embeddings of $E$ into $\operatorname{End}\left(\boldsymbol{D}_{\mathbb{Q}}^{\prime}\right)$ are conjugate, by the Noether-Skolem theorem, and so $\varrho$ may be modified to make it $E$-linear. Another application of Noether-Skolem shows that $\varrho$ may be further modified to ensure that the polarizations on $\boldsymbol{D}_{\mathbb{Q}}$ and $\boldsymbol{D}_{\mathbb{Q}}^{\prime}$ induce the same Rosati involution on

$$
\operatorname{End}\left(\boldsymbol{D}_{\mathbb{Q}}\right) \cong \operatorname{End}\left(\boldsymbol{D}_{\mathbb{Q}}^{\prime}\right)
$$

This implies that $\varrho$ identifies the polarizations, and hence the Hermitian forms, on $\boldsymbol{D}_{\mathbb{Q}}$ and $\boldsymbol{D}_{\mathbb{Q}}^{\prime}$ up to scaling by an element $c(\varrho) \in \mathbb{Q}_{p}^{\times}$.

Finally, for every $c \in \mathbb{Q}_{p}^{\times}$one can find an $E$-linear isocrystal automorphism $g$ of $\boldsymbol{D}_{\mathbb{Q}}^{\prime}$ such that $g$ rescales the polarization of $\boldsymbol{D}_{\mathbb{Q}}^{\prime}$ by the factor $c$. For example, if $\operatorname{ord}_{p}(c)$ is even then write $c=\alpha \bar{\alpha}$ with $\alpha \in E^{\times}$and take $g=\iota^{\prime}(\alpha)$. If $c=p$ then take $g$ to be

$$
\begin{aligned}
& e_{1} \mapsto e_{3}, \quad e_{2} \mapsto e_{4}, \quad e_{3} \mapsto p e_{1}, \quad e_{4} \mapsto p e_{2}, \\
& f_{1} \mapsto p f_{3}, \quad f_{2} \mapsto p f_{4}, \quad f_{3} \mapsto f_{1}, \quad f_{4} \mapsto f_{2} .
\end{aligned}
$$


Thus $\varrho$ may be further modified to ensure that $c(\varrho)=1$.

Lemma 2.2. There is an $\mathcal{O}_{E} \otimes W$-module generator $\omega \in \bigwedge_{E}^{4} \boldsymbol{D}$ such that $\langle\boldsymbol{\omega}, \boldsymbol{\omega}\rangle=1$ and $F \boldsymbol{\omega}=p^{2} \omega$. If $\omega^{\prime} \in \bigwedge_{E}^{4} \boldsymbol{D}$ is another such element, there is an $\alpha \in \mathcal{O}_{E}^{\times}$such that $\alpha \bar{\alpha}=1$ and $\omega^{\prime}=\alpha \omega$.

Proof. The $W$-module decomposition $\boldsymbol{D}=\epsilon_{0} \boldsymbol{D} \oplus \epsilon_{1} \boldsymbol{D}$ induces a corresponding decomposition $\bigwedge_{E}^{4} \boldsymbol{D}=\bigwedge^{4} \epsilon_{0} \boldsymbol{D} \oplus \wedge^{4} \epsilon_{1} \boldsymbol{D}$. Fixing a basis as in Lemma 2.1, we must have

$$
\begin{aligned}
& \wedge^{4} \epsilon_{0} \boldsymbol{D}=W \cdot p^{k_{0}} e_{1} \wedge e_{2} \wedge e_{3} \wedge e_{4}, \\
& \wedge^{4} \epsilon_{1} \boldsymbol{D}=W \cdot p^{k_{1}} f_{1} \wedge f_{2} \wedge f_{3} \wedge f_{4},
\end{aligned}
$$

for some integers $k_{0}$ and $k_{1}$. The self-duality of $\bigwedge_{E}^{4} \boldsymbol{D}$ under $\langle\cdot, \cdot\rangle$ implies $k_{0}+k_{1}=0$. The signature- $(2,2)$ condition on

$$
\operatorname{Lie}(\boldsymbol{G}) \cong \boldsymbol{D} / V \boldsymbol{D}=\epsilon_{0} \boldsymbol{D} / V \epsilon_{1} \boldsymbol{D} \oplus \epsilon_{1} \boldsymbol{D}_{1} / V \epsilon_{0} \boldsymbol{D}
$$

implies that each of the summands on the right has dimension 2 over $W / p W$, and hence the cokernels of

$$
V: \bigwedge^{4} \epsilon_{0} \boldsymbol{D} \rightarrow \wedge^{4} \epsilon_{1} \boldsymbol{D}, \quad V: \bigwedge^{4} \epsilon_{1} \boldsymbol{D} \rightarrow \bigwedge^{4} \epsilon_{0} \boldsymbol{D}
$$

are each of length 2 as $W$-modules. Using

$$
\begin{aligned}
V\left(e_{1} \wedge e_{2} \wedge e_{3} \wedge e_{4}\right) & =p^{2} f_{1} \wedge f_{2} \wedge f_{3} \wedge f_{4}, \\
V\left(f_{1} \wedge f_{2} \wedge f_{3} \wedge f_{4}\right) & =p^{2} e_{1} \wedge e_{2} \wedge e_{3} \wedge e_{4}
\end{aligned}
$$

we deduce that $k_{1}$ and $k_{2}$ are equal, and hence both are equal to 0 . It follows that

$$
\boldsymbol{\omega}=e_{1} \wedge e_{2} \wedge e_{3} \wedge e_{4}+f_{1} \wedge f_{2} \wedge f_{3} \wedge f_{4}
$$

generates $\bigwedge_{E}^{4} \boldsymbol{D}$ as an $\mathcal{O}_{E} \otimes W$-module. A simple calculation shows that $\langle\boldsymbol{\omega}, \boldsymbol{\omega}\rangle=1$ and $F \boldsymbol{\omega}=p^{2} \boldsymbol{\omega}$, proving the existence part of the lemma. The uniqueness part of the claim is obvious.

Definition 2.3. For any $\omega$ as in the lemma, define the Hodge star operator $x \mapsto x^{\star}$ on $\wedge_{E}^{2} \boldsymbol{D}$ by the relation $y \wedge x^{\star}=\langle y, x\rangle \cdot \omega$ for all $y \in \wedge_{E}^{2} \boldsymbol{D}$.

The Hodge operator satisfies $(\alpha x)^{\star}=\bar{\alpha} x^{\star}$ for all $\alpha \in \mathcal{O}_{E} \otimes W$. Denote by

$$
\boldsymbol{L}=\left\{x \in \bigwedge_{E}^{2} \boldsymbol{D}: x^{\star}=x\right\}
$$

the $W$-submodule of Hodge fixed vectors. The Hermitian form $\langle\cdot, \cdot\rangle$ on $\boldsymbol{D}$ determines an injection $\bigwedge_{E}^{2} \boldsymbol{D} \rightarrow \operatorname{End}_{W}(\boldsymbol{D})$ by

$$
(a \wedge b)(z)=\langle a, z\rangle b-\langle b, z\rangle a,
$$


and we obtain inclusions $\boldsymbol{L} \subset \bigwedge_{E}^{2} \boldsymbol{D} \subset \operatorname{End}_{W}(\boldsymbol{D})$. Note that both the Hodge star operator and the submodule $\boldsymbol{L}$ depend on the choice of $\boldsymbol{\omega}$.

Proposition 2.4. For any choice of $\omega$, the induced Hodge star operator has the following properties:

(1) Every $x \in \bigwedge_{E}^{2} \boldsymbol{D}$ satisfies $\left(x^{\star}\right)^{\star}=x$.

(2) Every $x \in \boldsymbol{L}$, viewed as an endomorphism of $\boldsymbol{D}$, satisfies

$$
x \circ x=-\frac{\langle x, x\rangle}{2} .
$$

In particular, $Q(x)=x \circ x$ defines a $W$-valued quadratic form on $\boldsymbol{L}$.

(3) The $W$-quadratic space $\boldsymbol{L}$ is self-dual of rank 6 , and

$$
\boldsymbol{L}=\left\{x \in \boldsymbol{L}_{\mathbb{Q}}: x \boldsymbol{D} \subset \boldsymbol{D}\right\} .
$$

(4) If $C(\boldsymbol{L})$ denotes the Clifford algebra of $\boldsymbol{L}$, the natural map

$$
C(\boldsymbol{L}) \rightarrow \operatorname{End}_{W}(\boldsymbol{D})
$$

induced by the inclusion $\boldsymbol{L} \subset \operatorname{End}_{W}(\boldsymbol{D})$ is an isomorphism. Under this isomorphism, the even Clifford algebra is identified with the subalgebra of $\mathcal{O}_{E}$-linear endomorphisms in $\operatorname{End}_{W}(\boldsymbol{D})$.

Proof. Fix a basis of $\boldsymbol{D}_{\mathbb{Q}}$ as in Lemma 2.1, and suppose first that $\boldsymbol{\omega}$ is given by (2-5). An easy calculation shows that

$$
\begin{array}{ll}
\left(e_{1} \wedge e_{2}\right)^{\star}=f_{3} \wedge f_{4}, & \left(f_{3} \wedge f_{4}\right)^{\star}=e_{1} \wedge e_{2}, \\
\left(e_{1} \wedge e_{3}\right)^{\star}=f_{4} \wedge f_{2}, & \left(f_{4} \wedge f_{2}\right)^{\star}=e_{1} \wedge e_{3}, \\
\left(e_{1} \wedge e_{4}\right)^{\star}=f_{2} \wedge f_{3}, & \left(f_{2} \wedge f_{3}\right)^{\star}=e_{1} \wedge e_{4}, \\
\left(e_{2} \wedge e_{3}\right)^{\star}=f_{1} \wedge f_{4}, & \left(f_{1} \wedge f_{4}\right)^{\star}=e_{2} \wedge e_{3}, \\
\left(e_{2} \wedge e_{4}\right)^{\star}=f_{3} \wedge f_{1}, & \left(f_{3} \wedge f_{1}\right)^{\star}=e_{2} \wedge e_{4}, \\
\left(e_{3} \wedge e_{4}\right)^{\star}=f_{1} \wedge f_{2}, & \left(f_{1} \wedge f_{2}\right)^{\star}=e_{3} \wedge e_{4},
\end{array}
$$

from which $\left(x^{\star}\right)^{\star}=x$ is obvious. Now set $\omega^{\prime}=\alpha \omega$ with $\alpha \in \mathcal{O}_{E}^{\times}$of norm 1 , and denote by $x \mapsto x^{\star \prime}$ the Hodge star operator defined by $\omega^{\prime}$. It is related to the Hodge star operator for $\omega$ by $x^{\star \prime}=\alpha x^{\star}$, and hence

$$
\left(x^{\star \prime}\right)^{\star \prime}=\left(\alpha\left(\alpha x^{\star}\right)\right)^{\star}=\alpha \bar{\alpha}\left(x^{\star}\right)^{\star}=x .
$$

This proves the first claim in full generality.

Keep $\omega$ as in (2-5). For the second claim, one first checks that all $x, y \in \bigwedge_{E}^{2} D$ satisfy the relation

$$
x \circ y+y^{\star} \circ x^{\star}=-\langle x, y\rangle
$$


in $\operatorname{End}_{W}(\boldsymbol{D})$. Indeed, it suffices to prove this when $x$ and $y$ are pure tensors of the form $e_{i} \wedge e_{j}$ and $f_{i} \wedge f_{j}$, and this can be done by brute force. Of course (2-8) immediately implies (2-6) for all $x \in \boldsymbol{L}$, proving the second claim for $\boldsymbol{\omega}$. The validity of (2-8) for any other $\omega^{\prime}$ follows by the reasoning of the previous paragraph.

For the third claim, note that the quadratic form $Q(x)=-\langle x, x\rangle / 2$ on $\boldsymbol{L}$ extends to a quadratic form on $\bigwedge_{E}^{2} \boldsymbol{D}$ by the same formula (using the standing hypothesis that $p$ is odd), with associated bilinear form

$$
[x, y]=-\frac{1}{2} \cdot \operatorname{Tr}_{E / \mathbb{Q}_{p}}\langle x, y\rangle,
$$

and that there is an orthogonal decomposition

$$
\bigwedge_{E}^{2} \boldsymbol{D}=\boldsymbol{L} \oplus\left\{x \in \bigwedge_{E}^{2} \boldsymbol{D}: x^{\star}=-x\right\} .
$$

The self-duality of $\bigwedge_{E}^{2} \boldsymbol{D}$ under $\langle\cdot, \cdot\rangle$ implies its self-duality under $[\cdot, \cdot]$, which then implies the self-duality of the orthogonal summand $\boldsymbol{L}$. The Hodge star operator acts on the $W$-module

$$
\bigwedge_{E}^{2} \boldsymbol{D}=\bigwedge^{2} \epsilon_{0} D \oplus \bigwedge^{2} \epsilon_{1} \boldsymbol{D}
$$

of rank 12 by interchanging the two summands on the right, and hence its submodule of fixed points, $\boldsymbol{L}$, has rank 6. Finally, set $\boldsymbol{L}^{\prime}=\left\{x \in \boldsymbol{L}_{\mathbb{Q}}: x \boldsymbol{D} \subset \boldsymbol{D}\right\}$. Certainly $\boldsymbol{L} \subset \boldsymbol{L}^{\prime}$, and the quadratic form $Q(x)=x \circ x$ restricted to $\boldsymbol{L}^{\prime}$ takes values in $W=W_{\mathbb{Q}} \cap \operatorname{End}_{W}(\boldsymbol{D})$. Therefore $\left(\boldsymbol{L}^{\prime}\right)^{\vee} \subset \boldsymbol{L}^{\vee}=\boldsymbol{L} \subset \boldsymbol{L}^{\prime} \subset\left(\boldsymbol{L}^{\prime}\right)^{\vee}$, and so equality holds throughout.

For the fourth claim, the self-duality of $\boldsymbol{L}$ implies that $\boldsymbol{L} / p \boldsymbol{L}$ is the unique nondegenerate $k$-quadratic space of dimension 6 , and so its Clifford algebra is isomorphic to $M_{8}(k)$. This means that the induced map

$$
C(\boldsymbol{L} / p \boldsymbol{L}) \cong C(\boldsymbol{L}) \otimes_{W} k \rightarrow \operatorname{End}_{W}(\boldsymbol{D}) \otimes_{W} k
$$

is a homomorphism between central simple $k$-algebras of the same dimension, and hence an isomorphism. It follows from Nakayama's lemma that $C(\boldsymbol{L}) \rightarrow \operatorname{End}_{W}(\boldsymbol{D})$ is an isomorphism. Every $x \in \boldsymbol{L}$ satisfies $x \circ \boldsymbol{\iota}(\alpha)=\boldsymbol{\iota}(\bar{\alpha}) \circ x$, and hence the composition of any two elements of $\boldsymbol{L}$ is $\mathcal{O}_{E}$-linear. This implies that the even Clifford algebra is contained in $\operatorname{End}_{\mathcal{O}_{E} \otimes W}(\boldsymbol{D})$, and equality holds because both are $W$-module direct summands of $C(\boldsymbol{L}) \cong \operatorname{End}_{W}(\boldsymbol{D})$ of the same rank.

The operator

$$
\Phi(a \wedge b)=p^{-1}(F a) \wedge(F b)
$$

makes $\bigwedge_{E}^{2} \boldsymbol{D}_{\mathbb{Q}}$ into a slope-0 isocrystal. In terms of the inclusion $\bigwedge_{E}^{2} \boldsymbol{D}_{\mathbb{Q}} \subset$ $\operatorname{End}_{W}(\boldsymbol{D})_{\mathbb{Q}}$, this operator is just

$$
\Phi(a \wedge b)=F \circ(a \wedge b) \circ F^{-1} .
$$


As $\Phi$ commutes with the Hodge star operator, it stabilizes the subspace $\boldsymbol{L}_{\mathbb{Q}}$ and makes $\boldsymbol{L}_{\mathbb{Q}}$ into a slope-0 isocrystal. In this way, we obtain inclusions of $\mathbb{Q}_{p}$-vector spaces

$$
\boldsymbol{L}_{\mathbb{Q}}^{\Phi} \subset\left(\bigwedge_{E}^{2} \boldsymbol{D}_{\mathbb{Q}}\right)^{\Phi} \subset \operatorname{End}(\boldsymbol{G})_{\mathbb{Q}},
$$

where the $\Phi$ superscripts denote the subspaces of $\Phi$-fixed vectors. Endow $\boldsymbol{L}_{\mathbb{Q}}^{\Phi}$ with the quadratic form $Q(x)=x \circ x$ and the associated bilinear form

$$
[x, y]=x \circ y+y \circ x=-\frac{1}{2} \cdot \operatorname{Tr}_{E / \mathbb{Q}_{p}}\langle x, y\rangle .
$$

Remark 2.5. The 6-dimensional $E$-vector space $\left(\bigwedge_{E}^{2} \boldsymbol{D}_{\mathbb{Q}}\right)^{\Phi}$ is characterized as the space of all Rosati-fixed $x \in \operatorname{End}(G)_{\mathbb{Q}}$ satisfying $x \circ \iota(\alpha)=\iota(\bar{\alpha}) \circ x$ for all $\alpha \in E$. On the other hand, the 6-dimensional $\mathbb{Q}_{p}$-vector space $L_{\mathbb{Q}}^{\Phi}$ depends on the choice of $\omega$, and so does not have a similar interpretation in terms of $\lambda$ and $\iota$ alone.

While the subspace $\boldsymbol{L}_{\mathbb{Q}}^{\Phi} \subset \operatorname{End}(\boldsymbol{G})_{\mathbb{Q}}$ depends on the choice of $\boldsymbol{\omega}$, the following proposition shows that its isomorphism class as a quadratic space does not. Denote by $\mathbb{W}$ the hyperbolic $\mathbb{Q}_{p}$-quadratic space of dimension 2 .

Proposition 2.6. For any choice of $\boldsymbol{\omega}$, the quadratic space $\boldsymbol{L}_{\mathbb{Q}}^{\Phi}$ has Hasse invariant -1 and determinant $\operatorname{det}\left(\boldsymbol{L}_{\mathbb{Q}}^{\Phi}\right)=-\Delta$ for any nonsquare $\Delta \in \mathbb{Z}_{p}^{\times}$. Furthermore, the special orthogonal group $\mathrm{SO}\left(\boldsymbol{L}_{\mathbb{Q}}^{\Phi}\right)$ is quasi-split and splits over $\mathbb{Q}_{p^{2}}$, and the space $\boldsymbol{L}_{\mathbb{Q}}^{\Phi}$ with the rescaled quadratic form $p^{-1} Q$ is isomorphic to $\mathbb{U}^{2} \oplus \mathbb{Q}_{p^{2}}$, where $\mathbb{Q}_{p^{2}}$ is endowed with its norm form $x \mapsto \operatorname{Norm}_{\mathbb{Q}_{p^{2}} / \mathbb{Q}_{p}}(x)$.

Proof. First suppose that $\omega$ is defined by (2-5). In this case the relations (2-7) show that the vectors

$$
\begin{array}{ll}
x_{1}=e_{1} \wedge e_{2}+f_{3} \wedge f_{4}, & x_{2}=e_{3} \wedge e_{4}+f_{1} \wedge f_{2}, \\
x_{3}=e_{1} \wedge e_{3}+f_{4} \wedge f_{2}, & x_{4}=e_{4} \wedge e_{2}+f_{1} \wedge f_{3}, \\
x_{5}=e_{1} \wedge e_{4}+f_{2} \wedge f_{3}, & x_{6}=e_{2} \wedge e_{3}+f_{1} \wedge f_{4}
\end{array}
$$

form a basis of $\boldsymbol{L}_{\mathbb{Q}}$. In this basis the operator $\Phi$ takes the block-diagonal form

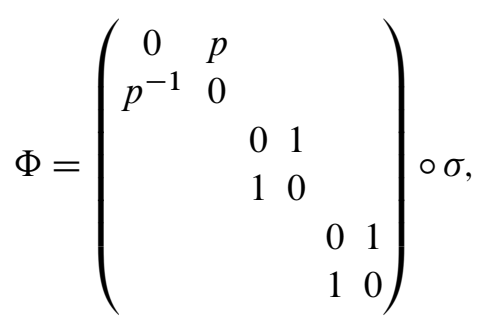


and the matrix of $Q$ is

$$
\left(\left[x_{i}, x_{j}\right]\right)=\left(\begin{array}{rrrrrr}
0 & -1 & & & & \\
-1 & 0 & & & & \\
& & 0 & -1 & & \\
& & -1 & 0 & & \\
& & & & 0 & -1 \\
& & & & -1 & 0
\end{array}\right) .
$$

Fix any nonsquare $\Delta \in \mathbb{Z}_{p}^{\times}$, and let $u \in W^{\times}$be a square root of $\Delta$. The vectors

$$
\begin{array}{ll}
y_{1}=p x_{1}+x_{2}, & y_{2}=u\left(p x_{1}-x_{2}\right), \\
y_{3}=x_{3}+x_{4}, & y_{4}=u\left(x_{3}-x_{4}\right), \\
y_{5}=x_{5}+x_{6}, & y_{6}=u\left(x_{5}-x_{6}\right)
\end{array}
$$

form an orthogonal basis of $\boldsymbol{L}_{\mathbb{Q}}^{\Phi}$ with

$$
\left(\frac{\left[y_{i}, y_{j}\right]}{2}\right)=\left(\begin{array}{llllll}
-p & & & & & \\
& p \Delta & & & & \\
& & -1 & & & \\
& & & \Delta & & \\
& & & & -1 & \\
& & & & & \Delta
\end{array}\right) \text {, }
$$

from which one computes the determinant $-\Delta$ and Hasse invariant $(-p, p \Delta)=-1$ of $\boldsymbol{L}_{\mathbb{Q}}^{\Phi}$. As a nondegenerate quadratic space over $\mathbb{Q}_{p}$ is determined by its rank, determinant, and Hasse invariant, the remaining claims are easily checked for this special choice of $\omega$.

Now suppose $\omega^{\prime}=\alpha \omega$ for some $\alpha \in \mathcal{O}_{E}^{\times}$of norm 1. Hilbert's Theorem 90 implies that there is some $\eta \in \mathcal{O}_{E}^{\times}$satisfying $\eta \bar{\eta}^{-1}=\alpha$. Denote by $x \mapsto x^{\star \prime}$ the Hodge star operator defined by $\boldsymbol{\omega}^{\prime}$, by $\boldsymbol{L}^{\prime} \subset \bigwedge_{E}^{2} \boldsymbol{D}$ the submodule of Hodge fixed vectors, and by $Q^{\prime}$ the quadratic form $x \circ x$ on $\boldsymbol{L}^{\prime}$. Using the relation $x^{\star \prime}=\alpha x^{\star}$, it is easy to see that the function $x \mapsto \eta x$ defines an isomorphism of quadratic spaces

$$
\left(\boldsymbol{L}_{\mathbb{Q}}^{\Phi}, \eta \bar{\eta} Q\right) \cong\left(\boldsymbol{L}_{\mathbb{Q}}^{\prime \Phi}, Q^{\prime}\right)
$$

In particular, there is a basis of $\boldsymbol{L}_{\mathbb{Q}}^{\prime \Phi}$ such that the quadratic form $Q^{\prime}$ is given by $\eta \bar{\eta}$ times the matrix of (2-10). The Hasse invariant and determinant (modulo squares) of the matrix in (2-10) are unchanged if the matrix is multiplied by any element of $\mathbb{Z}_{p}^{\times}$, and so $\boldsymbol{L}_{\mathbb{Q}}^{\prime \Phi}$ has the same determinant and Hasse invariant as $\boldsymbol{L}_{\mathbb{Q}}^{\Phi}$.

From now on we fix, once and for all, any $\omega$ as in Lemma 2.2. 
2.3. An exceptional isomorphism. Define the unitary similitude group

$$
\mathrm{GU}\left(\boldsymbol{D}_{\mathbb{Q}}\right)=\left\{g \in \operatorname{Aut}_{E \otimes W}\left(\boldsymbol{D}_{\mathbb{Q}}\right): g^{*} \lambda=v(g) \lambda \text { for some } v(g) \in W_{\mathbb{Q}}^{\times}\right\},
$$

and set

$$
\operatorname{GU}^{0}\left(\boldsymbol{D}_{\mathbb{Q}}\right)=\left\{g \in \operatorname{GU}\left(\boldsymbol{D}_{\mathbb{Q}}\right): v(g)^{2}=\operatorname{det}(g)\right\} .
$$

The action $\bullet$ of $\mathrm{GU}\left(\boldsymbol{D}_{\mathbb{Q}}\right)$ on $\operatorname{End}_{W}(\boldsymbol{D})_{\mathbb{Q}}$ defined by $g \bullet x=g \circ x \circ g^{-1}$ leaves invariant the subspace $\bigwedge_{E}^{2} \boldsymbol{D}_{\mathbb{Q}}$, and satisfies

$$
g \bullet(a \wedge b)=v(g)^{-1} \cdot(g a) \wedge(g b) .
$$

Using this formula one checks that the action of the subgroup $\operatorname{GU}^{0}\left(\boldsymbol{D}_{\mathbb{Q}}\right)$ commutes with the Hodge star operator on $\bigwedge_{E}^{2} \boldsymbol{D}_{\mathbb{Q}}$, and so preserves the subspace $\boldsymbol{L}_{\mathbb{Q}}$.

The canonical involution $x \mapsto x^{\prime}$ on the Clifford algebra $C(\boldsymbol{L})$ is the unique $W$-linear endomorphism satisfying $\left(x_{1} \cdots x_{k}\right)^{\prime}=x_{k} \cdots x_{1}$ for all $x_{1}, \ldots, x_{k} \in \boldsymbol{L}$, and the spinor similitude group of $\boldsymbol{L}_{\mathbb{Q}}$ is

$$
\operatorname{GSpin}\left(\boldsymbol{L}_{\mathbb{Q}}\right)=\left\{g \in C_{0}(\boldsymbol{L})_{\mathbb{Q}}^{\times}: g \boldsymbol{L}_{\mathbb{Q}} g^{-1}=\boldsymbol{L}_{\mathbb{Q}} \text { and } g^{\prime} g \in W_{\mathbb{Q}}^{\times}\right\} .
$$

Here, $C_{0}(\boldsymbol{L})$ is the even Clifford algebra. From [Bass 1974] or [Shimura 2010] we have the exact sequence

$$
1 \longrightarrow W_{\mathbb{Q}}^{\times} \longrightarrow \operatorname{GSpin}\left(\boldsymbol{L}_{\mathbb{Q}}\right) \longrightarrow \mathrm{SO}\left(\boldsymbol{L}_{\mathbb{Q}}\right) \longrightarrow 1 .
$$

Proposition 2.7. There is an isomorphism

$$
\operatorname{GSpin}\left(\boldsymbol{L}_{\mathbb{Q}}\right) \cong \operatorname{GU}^{0}\left(\boldsymbol{D}_{\mathbb{Q}}\right)
$$

compatible with the action of both groups on $\boldsymbol{L}_{\mathbb{Q}}$. In particular, the action of $\mathrm{GU}^{0}\left(\boldsymbol{D}_{\mathbb{Q}}\right)$ on $\boldsymbol{L}_{\mathbb{Q}}$ determines an exact sequence

$$
1 \longrightarrow W_{\mathbb{Q}}^{\times} \longrightarrow \mathrm{GU}^{0}\left(\boldsymbol{D}_{\mathbb{Q}}\right) \stackrel{g \mapsto g \bullet}{\longrightarrow} \operatorname{SO}\left(\boldsymbol{L}_{\mathbb{Q}}\right) \longrightarrow 1 .
$$

Proof. By Proposition 2.4 the inclusion of $\boldsymbol{L}$ into $\operatorname{End}_{W}(\boldsymbol{D})$ induces an isomorphism $C(\boldsymbol{L}) \cong \operatorname{End}_{W}(\boldsymbol{D})$, under which $C_{0}(\boldsymbol{L}) \cong \operatorname{End}_{\mathcal{O}_{E} \otimes W}(\boldsymbol{D})$. We will prove that the induced isomorphism

$$
C_{0}(\boldsymbol{L})_{\mathbb{Q}}^{\times} \cong \operatorname{Aut}_{E \otimes W}\left(\boldsymbol{D}_{\mathbb{Q}}\right)
$$

restricts to an isomorphism (2-12). Note that every element $x \in \boldsymbol{L}$, viewed as an endomorphism of $\boldsymbol{D}$, satisfies $\langle x a, b\rangle=-\overline{\langle a, x b\rangle}$ (indeed, this already holds for every $\left.x \in \bigwedge_{E}^{2} \boldsymbol{D}\right)$. Thus $\langle g a, b\rangle=\left\langle a, g^{\prime} b\right\rangle$ for every $g \in C_{0}(\boldsymbol{L})$ and $a, b \in \boldsymbol{D}$.

One inclusion of (2-12) is obvious: if $g \in \mathrm{GU}^{0}\left(\boldsymbol{D}_{\mathbb{Q}}\right)$ then, as noted above, the conjugation action of $g$ on $C(\boldsymbol{L})_{\mathbb{Q}} \cong \operatorname{End}_{W}(\boldsymbol{D})_{\mathbb{Q}}$ preserves the subspace $\boldsymbol{L}_{\mathbb{Q}}$. The relation $\langle g a, g b\rangle=\left\langle a, g^{\prime} g b\right\rangle$ implies that $v(g)=g^{\prime} g$, and so $g \in \operatorname{GSpin}\left(\boldsymbol{L}_{\mathbb{Q}}\right)$. 
For the other inclusion, start with a $g \in \mathrm{GSpin}\left(\boldsymbol{L}_{\mathbb{Q}}\right)$. The relation $\langle g a, g b\rangle=$ $\left(g^{\prime} g\right)\langle a, b\rangle$ shows that $g \in \mathrm{GU}\left(\boldsymbol{D}_{\mathbb{Q}}\right)$. To show that $v(g)^{2}=\operatorname{det}(g)$, fix any $x \in \boldsymbol{L}$ and any $y \in \bigwedge_{E}^{2} \boldsymbol{D}$ for which $\langle y, x\rangle \neq 0$. As $g \bullet x=g x g^{-1}$ lies in $\boldsymbol{L}_{\mathbb{Q}}$ by assumption, the Hodge star operator fixes $g \bullet x$. Thus

$$
(g \bullet y) \wedge(g \bullet x)=\langle g \bullet y, g \bullet x\rangle \boldsymbol{\omega}=\langle y, x\rangle \boldsymbol{\omega},
$$

where the second equality follows from (2-11). On the other hand, the Hodge star operator fixes $x$, and so

$$
(g \bullet y) \wedge(g \bullet x)=v(g)^{-2} \operatorname{det}(g)(y \wedge x)=v(g)^{-2} \operatorname{det}(g)\langle y, x\rangle \omega .
$$

This proves that $g \in \mathrm{GU}^{0}\left(\boldsymbol{D}_{\mathbb{Q}}\right)$, and completes the proof of (2-12).

The similitude character $v: \mathrm{GU}^{0}\left(\boldsymbol{D}_{\mathbb{Q}}\right) \rightarrow W_{\mathbb{Q}}^{\times}$restricts to $x \mapsto x^{2}$ on the subgroup $W_{\mathbb{Q}}^{\times}$, and so descends to the spinor norm

$$
\tilde{v}: \operatorname{SO}\left(\boldsymbol{L}_{\mathbb{Q}}\right) \rightarrow W_{\mathbb{Q}}^{\times} /\left(W_{\mathbb{Q}}^{\times}\right)^{2} .
$$

Remark 2.8. The group $J$ defined in Section 2.1 is characterized by

$$
J=\left\{g \in \mathrm{GU}\left(\boldsymbol{D}_{\mathbb{Q}}\right): g \circ F=F \circ g\right\},
$$

and we define a subgroup

$$
J^{0}=\left\{g \in \operatorname{GU}^{0}\left(\boldsymbol{D}_{\mathbb{Q}}\right): g \circ F=F \circ g\right\} .
$$

The isomorphism (2-12) restricts to an isomorphism $\operatorname{GSpin}\left(\boldsymbol{L}_{\mathbb{Q}}^{\Phi}\right) \cong J^{0}$, and hence there is an exact sequence

$$
1 \longrightarrow \mathbb{Q}_{p}^{\times} \longrightarrow J^{0} \longrightarrow \mathrm{SO}\left(L_{\mathbb{Q}}^{\Phi}\right) \longrightarrow 1
$$

which identifies $J^{\text {der }}$ with $\operatorname{Spin}\left(\boldsymbol{L}_{\mathbb{Q}}^{\Phi}\right)$. See [Knus et al. 1998, Proposition IV.15.27] for similar exceptional isomorphisms.

2.4. Dieudonné lattices and special lattices. In this subsection we show that the $k$-points of $\mathscr{N}$ can be identified with the set of homothety classes of certain lattices in $\boldsymbol{D}_{\mathbb{Q}}$, which we call Dieudonné lattices. We then use the inclusion

$$
\boldsymbol{L}_{\mathbb{Q}} \subset \operatorname{End}_{W}\left(\boldsymbol{D}_{\mathbb{Q}}\right)
$$

to construct a bijection between the set of homothety classes of Dieudonné lattices and a set of special lattices in the slope-0 isocrystal $\boldsymbol{L}_{\mathbb{Q}}$. Thus the points of $\mathscr{N}(k)$ are parametrized by these special lattices.

In fact, the proof of Theorem 3.9 below requires that we establish such a bijection not just over $k$, but over any extension field $k^{\prime} \supset k$. Let $W^{\prime}$ be the Cohen ring of $k^{\prime}$. Thus $W^{\prime}$ is the unique, up to isomorphism, complete discrete valuation ring of mixed characteristic with residue field $W^{\prime} / p W^{\prime} \cong k^{\prime}$. The inclusion $k \rightarrow k^{\prime}$ 
induces an injective ring homomorphism $W \rightarrow W^{\prime}$, and we set $\boldsymbol{D}^{\prime}=\boldsymbol{D} \otimes_{W} W^{\prime}$ and $\boldsymbol{L}^{\prime}=\boldsymbol{L} \otimes_{W} W^{\prime}$. There is a continuous ring homomorphism $\sigma: W^{\prime} \rightarrow W^{\prime}$ reducing to the Frobenius on $k^{\prime}$, and the $\sigma$-semilinear operators $F$ and $\Phi$ on $\boldsymbol{D}_{\mathbb{Q}}$ and $\boldsymbol{L}_{\mathbb{Q}}$ have $\sigma$-semilinear extensions to $\boldsymbol{D}_{\mathbb{Q}}^{\prime}$ and $\boldsymbol{L}_{\mathbb{Q}}^{\prime}$. Similarly the symplectic and Hermitian forms on $\boldsymbol{D}_{\mathbb{Q}}$ and the quadratic form on $\boldsymbol{L}_{\mathbb{Q}}$ have natural extensions to $\boldsymbol{D}_{\mathbb{Q}}^{\prime}$ and $\boldsymbol{L}_{\mathbb{Q}}^{\prime}$.

Note that the operators $F$ and $\Phi$ are surjective on $\boldsymbol{D}_{\mathbb{Q}}$ and $\boldsymbol{L}_{\mathbb{Q}}$, respectively, but this need not be true of their extensions to $\boldsymbol{D}_{\mathbb{Q}}^{\prime}$ and $\boldsymbol{L}_{\mathbb{Q}}^{\prime}$. If $D \subset \boldsymbol{D}_{\mathbb{Q}}^{\prime}$ is a $W^{\prime}$-submodule then so is its preimage $F^{-1}(D)$, but its image $F(D)$ need not be. Denote by $F_{*}(D)$ the $W^{\prime}$-submodule generated by $F(D)$. Similarly, denote by $\Phi_{*}(L)$ the $W^{\prime}$-submodule generated by $\Phi(L)$ for a $W^{\prime}$-submodule $L \subset \boldsymbol{L}_{\mathbb{Q}}^{\prime}$.

For any $W^{\prime}$-lattice $D \subset \boldsymbol{D}_{\mathbb{Q}}^{\prime}$, set $D_{1}=F^{-1}(p D)$.

Definition 2.9. A Dieudonné lattice in $\boldsymbol{D}_{\mathbb{Q}}^{\prime}$ is an $\mathcal{O}_{E}$-stable $W^{\prime}$-lattice $D \subset \boldsymbol{D}_{\mathbb{Q}}^{\prime}$ such that

(1) $p D \subset D_{1} \subset D$,

(2) $D^{\vee}=c D$ for some $c \in \mathbb{Q}_{p}^{\times}$,

(3) $D=F_{*}\left(F^{-1}(D)\right)$.

Here, the superscript $\vee$ denotes the dual lattice with respect to the symplectic form $\lambda$, or, equivalently, with respect to the Hermitian form $\langle\cdot, \cdot\rangle$.

The volume of a lattice $D \subset \boldsymbol{D}_{\mathbb{Q}}^{\prime}$ is the $W^{\prime}$-submodule

$$
\operatorname{Vol}(D)=\wedge^{8} D \subset \wedge^{8} \boldsymbol{D}_{\mathbb{Q}}^{\prime},
$$

By considering the slopes of the isocrystal $\boldsymbol{D}_{\mathbb{Q}}$, one can show that $\operatorname{Vol}\left(F_{*}(D)\right)=$ $p^{4} \cdot \operatorname{Vol}(D)$. However, taking preimages of lattices may change volumes in unexpected ways: a lattice $D \subset \boldsymbol{D}_{\mathbb{Q}}^{\prime}$ satisfies

$$
\operatorname{Vol}\left(F^{-1}(D)\right) \subset p^{-4} \cdot \operatorname{Vol}(D),
$$

but equality holds if and only if $F_{*}\left(F^{-1}(D)\right)=D$. In particular, the condition $D=F_{*}\left(F^{-1}(D)\right)$ in Definition 2.9 is equivalent to $\operatorname{Vol}\left(D_{1}\right)=p^{4} \operatorname{Vol}(D)$, and so one could replace (3) in the definition of a Dieudonné lattice by

$\left(3^{\prime}\right) \operatorname{dim}_{k^{\prime}}\left(D_{1} / p D\right)=4$.

The volume of a lattice in $\boldsymbol{L}_{\mathbb{Q}}^{\prime}$ is defined in the analogous way, but in this case $\operatorname{Vol}\left(\Phi_{*}(L)\right)=\operatorname{Vol}(L)$ for any lattice $L \subset \boldsymbol{L}_{\mathbb{Q}}^{\prime}$.

Proposition 2.10. Suppose $D$ is a Dieudonné lattice. The $\mathcal{O}_{E}$-stable $k^{\prime}$-subspace $D_{1} / p D \subset D / p D$ is Lagrangian with respect to the nondegenerate symplectic form $c \lambda$, and every $\alpha \in \mathcal{O}_{E}$ acts on $D / D_{1}$ with characteristic polynomial

$$
\operatorname{det}\left(T-\iota(\alpha) ; D / D_{1}\right)=\left(T-\psi_{0}(\alpha)\right)^{2}\left(T-\psi_{1}(\alpha)\right)^{2} .
$$


Proof. For any $a, b \in D_{1}$ we have

$$
c \lambda(a, b)^{\sigma}=p^{-1} c \lambda(F a, F b) \in p \lambda(c D, D)=p W .
$$

This shows that $D_{1} / p D$ is isotropic. It is maximal isotropic, as $D_{1} / p D$ has dimension 4. Lemma 2.1 implies that

$$
\bigwedge^{4} F_{*}\left(\epsilon_{0} M\right)=p^{2} \cdot \wedge^{4} \epsilon_{1} M,
$$

as submodules of $\wedge^{4} \epsilon_{1} \boldsymbol{D}_{\mathbb{Q}}^{\prime}$, for any lattice $M \subset \boldsymbol{D}_{\mathbb{Q}}^{\prime}$. Applying this with $M=D_{1}$ shows that $\epsilon_{1} D / \epsilon_{1} D_{1}$ has dimension 2. The same argument shows that $\epsilon_{0} D / \epsilon_{0} D_{1}$ has dimension 2 , and (2-13) follows.

Corollary 2.11. There is a bijection $\mathscr{M}\left(k^{\prime}\right) \cong\left\{\right.$ Dieudonné lattices in $\left.\boldsymbol{D}_{\mathbb{Q}}^{\prime}\right\}$.

Proof. If $k=k^{\prime}$ then this is immediate from the equivalence of categories between Dieudonné modules and $p$-divisible groups: for any point $(G, \iota, \lambda, \varrho) \in \mathscr{M}(k)$ we let $D$ be the Dieudonné module of $G$, viewed as a lattice in $\boldsymbol{D}_{\mathbb{Q}}$ using the isomorphism of isocrystals $\varrho: D_{\mathbb{Q}} \cong \boldsymbol{D}_{\mathbb{Q}}$. For general $k^{\prime}$ the argument is the same, using Zink's theory of windows [2001] in place of Dieudonné modules.

Theorem 2.12. Given a Dieudonné lattice D, set

$$
L=\left\{x \in \boldsymbol{L}_{\mathbb{Q}}^{\prime}: x D_{1} \subset D_{1}\right\} \quad \text { and } \quad L^{\sharp}=\left\{x \in \boldsymbol{L}_{\mathbb{Q}}^{\prime}: x D \subset D\right\} .
$$

The rule $D \mapsto\left(L, L^{\sharp}\right)$ defines a bijection from $p^{\mathbb{Z}} \backslash\left\{\right.$ Dieudonné lattices in $\left.\boldsymbol{D}_{\mathbb{Q}}^{\prime}\right\}$ to the set of all pairs of self-dual lattices $\left(L, L^{\#}\right)$ in $\boldsymbol{L}_{\mathbb{Q}}^{\prime}$ such that

(1) $\Phi_{*}(L)=L^{\sharp}$,

(2) $\left(L+L^{\sharp}\right) / L$ has length 1 .

Moreover, $L+L^{\sharp}=\left\{x \in \boldsymbol{L}_{\mathbb{Q}}^{\prime}: x D_{1} \subset D\right\}$.

The proof of Theorem 2.12 will be given in the next subsection.

Definition 2.13. A special lattice is a self-dual $W^{\prime}$-lattice $L \subset \boldsymbol{L}_{\mathbb{Q}}^{\prime}$ such that

$$
\text { length }\left(\left(L+\Phi_{*}(L)\right) / L\right)=1 \text {. }
$$

Obviously any pair of self-dual lattices $\left(L, L^{\sharp}\right)$ appearing in Theorem 2.12 is determined by its first element, and in fact the function $L \mapsto\left(L, \Phi_{*}(L)\right)$ establishes a bijection between the set of special lattices and the set of pairs of self-dual lattices $\left(L, L^{\sharp}\right)$ such that $\Phi_{*}(L)=L^{\sharp}$ and $\left(L+L^{\sharp}\right) / L$ has length 1 . The only thing to check is the self-duality of $\Phi_{*}(L)$ for a special lattice $L$. The inclusion $\Phi_{*}(L) \subset \Phi_{*}(L)^{\vee}$ is clear from the self-duality of $L$ and the relation $[\Phi x, \Phi y]=[x, y]^{\sigma}$. Equality holds because $\operatorname{Vol}\left(\Phi_{*}(L)\right)=\operatorname{Vol}(L)$ and $L$ is self-dual. The following corollary is now simply a restatement of Theorem 2.12: 
Corollary 2.14. The rule $D \mapsto\left\{x \in L_{\mathbb{Q}}^{\prime}: x D_{1} \subset D_{1}\right\}$ defines a bijection $p^{\mathbb{Z}} \backslash\left\{\right.$ Dieudonné lattices in $\left.\boldsymbol{D}_{\mathbb{Q}}^{\prime}\right\} \cong\left\{\right.$ special lattices in $\left.\boldsymbol{L}_{\mathbb{Q}}^{\prime}\right\}$.

2.5. Proof of Theorem 2.12. In this subsection we prove Theorem 2.12. Say that a $W^{\prime}$-lattice $D \subset \boldsymbol{D}_{\mathbb{Q}}^{\prime}$ is nearly self-dual if $D^{\vee}=c D$ for some $c \in \mathbb{Q}_{p}^{\times}$.

Lemma 2.15. The construction $D \mapsto\left\{x \in L_{\mathbb{Q}}^{\prime}: x D \subset D\right\}$ establishes a bijection $p^{\mathbb{Z}} \backslash\left\{\right.$ nearly self-dual lattices $\left.D \subset \boldsymbol{D}_{\mathbb{Q}}^{\prime}\right\} \cong\left\{\right.$ self-dual lattices $\left.L^{\sharp} \subset \boldsymbol{L}_{\mathbb{Q}}^{\prime}\right\}$.

Proof. Start with a nearly self-dual lattice $D$, and set $L^{\sharp}=\left\{x \in \boldsymbol{L}_{\mathbb{Q}}^{\prime}: x D \subset D\right\}$. The condition $D^{\vee}=c D$ implies that there is some $g \in \mathrm{GU}^{0}\left(\boldsymbol{D}_{\mathbb{Q}}^{\prime}\right)$ such that $D=g \boldsymbol{D}^{\prime}$, and hence $L^{\sharp}=g \bullet \boldsymbol{L}^{\prime}$. As $g \bullet$ respects the quadratic form $Q$, the self-duality of $\boldsymbol{L}^{\prime}$ implies the self-duality of $L^{\sharp}$. Conversely, if we start with a self-dual $L^{\sharp} \subset \boldsymbol{L}_{\mathbb{Q}}^{\prime}$, the Clifford algebra $C\left(L^{\sharp}\right)$ is a maximal order in $C\left(\boldsymbol{L}_{\mathbb{Q}}^{\prime}\right) \cong \operatorname{End}_{W^{\prime}}\left(\boldsymbol{D}_{\mathbb{Q}}^{\prime}\right)$, and so there is, up to scaling, a unique lattice $D \subset \boldsymbol{D}_{\mathbb{Q}}^{\prime}$ satisfying

$$
C\left(L^{\sharp}\right)=\operatorname{End}_{W}(D) .
$$

Choose any $h \in \operatorname{SO}\left(\boldsymbol{L}_{\mathbb{Q}}^{\prime}\right)$ such that $L^{\#}=h \boldsymbol{L}^{\prime}$, and lift $h$ to an element $g \in \operatorname{GU}^{0}\left(\boldsymbol{D}_{\mathbb{Q}}^{\prime}\right)$. By rescaling $g$ we may arrange to have $D=g \boldsymbol{D}^{\prime}$, and the self-duality of $\boldsymbol{D}^{\prime}$ implies $D^{\vee}=v(g)^{-1} D$.

Lemma 2.16. Suppose $D \subset D_{\mathbb{Q}}^{\prime}$ is nearly self-dual, $L^{\sharp} \subset \boldsymbol{L}_{\mathbb{Q}}^{\prime}$ is self-dual, and $L^{\sharp}$ and $D$ are related by $L^{\sharp}=\left\{x \in L_{\mathbb{Q}}^{\prime}: x D \subset D\right\}$. If $x \in L^{\sharp} / p L^{\sharp}$ is any nonzero isotropic vector, viewed as an endomorphism of $D / p D$ using (2-14), the kernel of $x$ is an $\mathcal{O}_{E}$-stable Lagrangian subspace with respect to $c \lambda$. Conversely, if $\mathscr{D}_{1} \subset D / p D$ is an $\mathcal{O}_{E}$-stable Lagrangian subspace then $\left\{x \in L^{\sharp} / p L^{\sharp}: x \mathscr{D}_{1}=0\right\}$ is an isotropic line in $L^{\sharp} / p L^{\sharp}$. This construction establishes a bijection

$\left\{\right.$ isotropic lines in $\left.L^{\sharp} / p L^{\sharp}\right\} \cong\left\{\mathcal{O}_{E}\right.$-stable Lagrangian subspaces in $\left.D / p D\right\}$. If $\mathscr{L}_{1} \subset L^{\sharp} / p L^{\sharp}$ corresponds to $\mathscr{D}_{1} \subset D / p D$ under this bijection, then

$$
\mathscr{L}_{1}^{\perp}=\left\{x \in L^{\sharp} / p L^{\sharp}: x \cdot \mathscr{D}_{1} \subset \mathscr{D}_{1}\right\} .
$$

Proof. Abbreviate $\mathscr{L}=L^{\sharp} / p L^{\sharp}$ and $\mathscr{D}=D / p D$, so that $\mathscr{D}$ is the unique simple left module over the Clifford algebra $C(\mathscr{L}) \cong M_{8}\left(k^{\prime}\right)$. In particular $C(\mathscr{L}) \cong \mathscr{D}^{8}$ as left $C(\mathscr{L})$-modules. If $x \in \mathscr{L}$ is any nonzero isotropic vector, the kernel and image of left multiplication by $x$ on $C(\mathscr{L})$ are equal, and hence the kernel and image of $x \in \operatorname{End}(\mathscr{D})$ are also equal. In particular $\operatorname{ker}(x)$ has dimension 4 . The relation $\alpha x=x \bar{\alpha}$ for all $\alpha \in \mathcal{O}_{E}$ shows that $\operatorname{ker}(x)$ is $\mathcal{O}_{E}$-stable, and the relation $(c \lambda)(x s, t)=(c \lambda)(s, x t)$ implies that $\operatorname{ker}(x)=x \mathscr{D}$ is totally isotropic.

If $x, y \in \mathscr{L}$ are nonzero isotropic vectors with $\operatorname{ker}(x)=\operatorname{ker}(y)$ then, from the discussion above, $\operatorname{ker}(x)=y \mathscr{D}$ and $\operatorname{ker}(y)=x \mathscr{D}$. In particular $[x, y]=$ 
$x \circ y+y \circ x=0$. If $x$ and $y$ are not colinear then (after possibly extending scalars) we can find a $z \in \mathscr{L}$ such that $k^{\prime} x+k^{\prime} y+k^{\prime} z$ is a maximal isotropic subspace of $\mathscr{L}$. The left ideal $C(\mathscr{L}) x y z$ has dimension 8 as a $k$-vector space, and so we must have $\mathscr{D} \cong C(\mathscr{L}) x y z$ as left $C(\mathscr{L})$-modules. But it is easy to see by direct calculation that the kernels of left multiplication by $x$ and $y$ on $C(\mathscr{L}) x y z$ are different. This contradiction shows that $x$ and $y$ are colinear, and so $x \mapsto \operatorname{ker}(x)$ establishes an injection $\mathscr{L}_{1} \mapsto \mathscr{D}_{1}$ from the set of isotropic lines in $\mathscr{L}$ to the set of $\mathcal{O}_{E}$-stable Lagrangian subspaces in $\mathscr{D}$.

Endow $\mathscr{D}$ with the $\mathcal{O}_{E} \otimes_{\mathbb{Z}_{p}} k^{\prime}$-valued Hermitian form induced by $c\langle\cdot, \cdot\rangle$. Exactly as in Proposition 2.7, there is an isomorphism of $k^{\prime}$-groups $\operatorname{GSpin}(\mathscr{L}) \cong \operatorname{GU}^{0}(\mathscr{D})$. This isomorphism is compatible, in the obvious sense, with the map $\mathscr{L}_{1} \mapsto \mathscr{D}_{1}$, and so the image of the map is stable under the action of $\mathrm{GU}^{0}(\mathscr{D})$. But $\mathrm{GU}^{0}(\mathscr{D})$ acts transitively on the set of $\mathcal{O}_{E}$-stable Lagrangian subspaces in $\mathscr{D}$, proving surjectivity.

Finally, we verify (2-15). If $\mathscr{L}_{1}$ corresponds to $\mathscr{D}_{1}$ under our bijection, then $\mathscr{D}_{1}=\operatorname{ker}(y)=y \mathscr{D}$ for any nonzero $y \in \mathscr{L}_{1}$, and an elementary argument (using $k^{\prime} \cap y C(\mathscr{L})=0$ for the middle $\Leftarrow$ ) shows that

$$
x \perp y \Longleftrightarrow x y+y x=0 \Longleftrightarrow x y C(\mathscr{L}) \subset y C(\mathscr{L}) \Longleftrightarrow x y \mathscr{D} \subset y \mathscr{D}_{1} .
$$

Proof of Theorem 2.12. Suppose first that $D$ is a Dieudonné lattice. Using the relations $D=F_{*}\left(F^{-1}(D)\right)$ and $\langle F v, F w\rangle=p\langle v, w\rangle^{\sigma}$, one can show that the near self-duality of $D$ implies that $D_{1}=F^{-1}(p D)$ is also nearly self-dual. Lemma 2.15 then implies that the lattices

$$
L=\left\{x \in \boldsymbol{L}_{\mathbb{Q}}^{\prime}: x D_{1} \subset D_{1}\right\} \quad \text { and } \quad L^{\sharp}=\left\{x \in \boldsymbol{L}_{\mathbb{Q}}^{\prime}: x D \subset D\right\}
$$

are self-dual. The relation $\Phi(x) \circ F=F \circ x$ for all $x \in \boldsymbol{L}_{\mathbb{Q}}^{\prime}$ implies that $\Phi_{*}(L) \subset L^{\sharp}$, and equality must hold as

$$
\operatorname{Vol}\left(\Phi_{*}(L)\right)=\operatorname{Vol}(L)=\operatorname{Vol}\left(L^{\sharp}\right) .
$$

By Proposition 2.10 the $k$-subspace $D_{1} / p D \subset D / p D$ is $\mathcal{O}_{E}$-stable and Lagrangian, and so it follows from Lemma 2.16 that

$$
\mathscr{L}_{1}=\left\{x \in L^{\sharp} / p L^{\sharp}: x\left(D_{1} / p D\right)=0\right\}
$$

is an isotropic line in $L^{\sharp} / p L^{\sharp}$ with orthogonal complement

$$
\mathscr{L}_{1}^{\perp}=\left\{x \in L^{\sharp} / p L^{\sharp}: x\left(D_{1} / p D\right) \subset D_{1} / p D\right\} .
$$

On the other hand, $L \cap L^{\sharp}=\left\{x \in L^{\sharp}: x D_{1} \subset D_{1}\right\}$, and so

$$
\left(L+L^{\sharp}\right) / L \cong L^{\sharp} /\left(L \cap L^{\sharp}\right) \cong\left(L^{\sharp} / p L^{\sharp}\right) / \mathscr{L}_{1}^{\perp}
$$

has length 1 . 
Now suppose we start with a pair of self-dual lattices $\left(L, L^{\sharp}\right)$ such that $\Phi_{*}(L)=$ $L^{\sharp}$ and $\left(L+L^{\sharp}\right) / L$ has length 1 . By Lemma 2.15 there are unique (up to scaling) nearly self-dual lattices $D_{1}$ and $D$ in $\boldsymbol{D}_{\mathbb{Q}}^{\prime}$ satisfying (2-16). Set $L_{0}=L \cap L^{\sharp}$, so that $L^{\sharp} / L_{0}$ has length 1 , and pick any nonzero $y \in L^{\sharp} / L_{0}$. The Clifford algebra $C\left(L^{\sharp}\right)$ satisfies

$$
C\left(L^{\sharp}\right)=C\left(L_{0}\right)+y C\left(L_{0}\right),
$$

where $C\left(L_{0}\right) \subset C\left(L^{\sharp}\right)$ is the $W$-subalgebra generated by $L_{0}$, and so

$$
C\left(L^{\sharp}\right) D_{1}=C\left(L_{0}\right) D_{1}+y C\left(L_{0}\right) D_{1}=D_{1}+y D_{1} .
$$

This implies $C\left(L^{\sharp}\right) p D_{1} \subset D_{1} \subset C\left(L^{\sharp}\right) D_{1}$. The self-duality of $L^{\sharp}$ implies that $C\left(L^{\sharp}\right)$ is a maximal order in $C\left(\boldsymbol{L}_{\mathbb{Q}}^{\prime}\right)=\operatorname{End}_{W^{\prime}}\left(\boldsymbol{D}_{\mathbb{Q}}^{\prime}\right)$, and so we must have $C\left(L^{\sharp}\right)=$ $\operatorname{End}_{W^{\prime}}(D)$. As the lattice $C\left(L^{\sharp}\right) D_{1}$ is obviously stabilized by $C\left(L^{\sharp}\right)$, it must have the form $C\left(L^{\sharp}\right) D_{1}=p^{k} D$ for some integer $k$. Thus after rescaling $D_{1}$ we may assume that $C\left(L^{\sharp}\right) D_{1}=D$ and

$$
p D \subset D_{1} \subset D
$$

The relation $\Phi(x) \circ F=F \circ x$ implies

$$
C\left(L^{\sharp}\right) F_{*}\left(D_{1}\right)=C\left(\Phi_{*}(L)\right) F_{*}\left(D_{1}\right)=F_{*}\left(C(L) D_{1}\right)=F_{*}\left(D_{1}\right),
$$

and so $F_{*}\left(D_{1}\right)=p^{k} D$ for some $k$. Combining

$$
p^{8 k} \cdot \operatorname{Vol}(D)=\operatorname{Vol}\left(F_{*}\left(D_{1}\right)\right)=p^{4} \cdot \operatorname{Vol}\left(D_{1}\right)
$$

and

$$
p^{8} \cdot \operatorname{Vol}(D) \subset \operatorname{Vol}\left(D_{1}\right) \subset \operatorname{Vol}(D)
$$

shows that in fact $F_{*}\left(D_{1}\right)=p D$. The relations $D_{1}=F^{-1}(p D)$ and $F_{*}\left(F^{-1}(D)\right)=$ $D$ follow easily from this, proving that $D$ is a Dieudonné lattice.

It only remains to prove that $L+L^{\sharp}=\left\{x \in L_{\mathbb{Q}}^{\prime}: x D_{1} \subset D\right\}$. The inclusion $L+L^{\sharp} \subset\left\{x \in L_{\mathbb{Q}}^{\prime}: x D_{1} \subset D\right\}$ is obvious from (2-16). On the other hand, each side contains $L^{\sharp}$ with quotient of length 1 (for the right-hand side this follows from Proposition 2.10 and Lemma 2.16). Thus equality holds.

2.6. Vertex lattices and the Bruhat-Tits stratification. If we start with a $k$-point $(G, \lambda, i, \varrho) \in \mathscr{M}(k)$ and let $D$ be the covariant Dieudonné module of $G$, then $\varrho(D) \subset \boldsymbol{D}_{\mathbb{Q}}$ is a Dieudonné lattice. This construction is simply the $k^{\prime}=k$ case of the bijection

$$
\mathscr{M}(k) \cong\left\{\text { Dieudonné lattices in } \boldsymbol{D}_{\mathbb{Q}}\right\}
$$

of Corollary 2.11. Combining this with Corollary 2.14 yields a bijection

$$
\mathscr{N}(k) \cong\left\{\text { special lattices in } \boldsymbol{L}_{\mathbb{Q}}\right\}
$$


defined by

$$
(G, \lambda, i, \varrho) \mapsto\left\{x \in \boldsymbol{L}_{\mathbb{Q}}: x \varrho\left(D_{1}\right) \subset \varrho\left(D_{1}\right)\right\},
$$

where $D_{1}=V D$. Moreover, Theorem 2.12 implies that the special lattice

$$
L=\left\{x \in \boldsymbol{L}_{\mathbb{Q}}: x \varrho\left(D_{1}\right) \subset \varrho\left(D_{1}\right)\right\}
$$

satisfies

$$
\Phi(L)=\left\{x \in \boldsymbol{L}_{\mathbb{Q}}: x \varrho(D) \subset \varrho(D)\right\} .
$$

The next step is to show that the special lattices come in natural families, indexed by certain vertex lattices in the $\mathbb{Q}_{p}$-quadratic space $\boldsymbol{L}_{\mathbb{Q}}^{\Phi}$. Using this and the bijection (2-17), we will then express the reduced scheme underlying $\mathscr{N}$ as a union of closed subvarieties indexed by vertex lattices.

Definition 2.17. A vertex lattice is a $\mathbb{Z}_{p}$-lattice $\Lambda \subset \boldsymbol{L}_{\mathbb{Q}}^{\Phi}$ such that

$$
p \Lambda \subset \Lambda^{\vee} \subset \Lambda \text {. }
$$

The type of $\Lambda$ is $t_{\Lambda}=\operatorname{dim}_{k}\left(\Lambda / \Lambda^{\vee}\right)$.

Lemma 2.18. The type of a vertex lattice is either 2,4 , or 6 .

Proof. Let $\Lambda$ be a vertex lattice. Proposition 2.6 implies that $\operatorname{ord}_{p}(\operatorname{det}(\Lambda))$ is even, from which it follows that the type of $\Lambda$ is also even. If $\Lambda$ has type 0 then $\Lambda$ is self-dual, and hence admits a basis such that the matrix of $Q$ is diagonal with diagonal entries in $\mathbb{Z}_{p}^{\times}$. But this implies that $\boldsymbol{L}_{\mathbb{Q}}^{\Phi}$ has Hasse invariant 1, contradicting Proposition 2.6.

The proof of the following proposition is identical to that of Proposition 4.1 of [Rapoport et al. 2014]. See also Lemma 2.1 of [Vollaard 2010].

Proposition 2.19. Let $L \subset \boldsymbol{L}_{\mathbb{Q}}$ be a special lattice, and define

$$
L^{(r)}=L+\Phi(L)+\cdots+\Phi^{r}(L) .
$$

There is an integer $d \in\{1,2,3\}$ such that

$$
L=L^{(0)} \subsetneq L^{(1)} \subsetneq \cdots \subsetneq L^{(d)}=L^{(d+1)} .
$$

For each $L^{(r)} \subsetneq L^{(r+1)}$ with $0 \leq r<d$, the quotient $L^{(r+1)} / L^{(r)}$ is annihilated by $p$ and satisfies $\operatorname{dim}_{k}\left(L^{(r+1)} / L^{(r)}\right)=1$. Moreover,

$$
\Lambda_{L}=\left\{x \in L^{(d)}: \Phi(x)=x\right\}
$$

is a vertex lattice of type $2 d$ and satisfies $\Lambda_{L}^{\vee}=\{x \in L: \Phi(x)=x\}$. 
By (2-9), each vertex lattice $\Lambda$ determines a collection of quasi-endomorphisms $\Lambda^{\vee} \subset \operatorname{End}(\boldsymbol{G})_{\mathbb{Q}}$. Define a closed formal subscheme $\widetilde{\mathscr{M}}_{\Lambda} \subset \mathscr{M}$ as the locus of points $(G, \iota, \lambda, \varrho)$ such that

$$
\varrho^{-1} \Lambda^{\vee} \varrho=\left\{\varrho^{-1} \circ x \circ \varrho: x \in \Lambda^{\vee}\right\} \subset \operatorname{End}(G) .
$$

In other words, the locus where the quasi-endomorphisms $\varrho^{-1} \Lambda^{\vee} \varrho$ of $G$ are actually integral. Set $\widetilde{\mathscr{N}}_{\Lambda}=p^{\mathbb{Z}} \backslash \widetilde{\mathscr{M}}_{\Lambda}$, and let $\mathscr{N}_{\Lambda}$ be the reduced $k$-scheme underlying $\tilde{\mathscr{N}}_{\Lambda}$. The bijection (2-17) identifies

$$
\begin{aligned}
\mathscr{N}_{\Lambda}(k) & =\left\{\text { special lattices } L \text { such that } \Lambda^{\vee} \subset \Phi(L)\right\} \\
& =\left\{\text { special lattices } L \text { such that } \Lambda^{\vee} \subset L\right\} \\
& =\left\{\text { special lattices } L \text { such that } \Lambda_{L} \subset \Lambda\right\} .
\end{aligned}
$$

The same proof used in [Rapoport et al. 2014, Proposition 4.3] shows that

$$
\mathscr{N}_{\Lambda_{1}} \cap \mathscr{N}_{\Lambda_{2}}= \begin{cases}\mathscr{N}_{\Lambda_{1} \cap \Lambda_{2}} & \text { if } \Lambda_{1} \cap \Lambda_{2} \text { is a vertex lattice, } \\ \varnothing & \text { otherwise, }\end{cases}
$$

where the left-hand side is understood to mean the reduced subscheme underlying the scheme-theoretic intersection.

Proposition 2.20. Each $k$-scheme $\mathscr{N}_{\Lambda}$ is projective.

Proof. Let $R_{\Lambda}$ be the $W$-subalgebra of $\operatorname{End}_{W}\left(\boldsymbol{D}_{\mathbb{Q}}\right)$ generated by $\Lambda^{\vee}$, and let $\widetilde{R}_{\Lambda}$ be a maximal order in $\operatorname{End}_{W}\left(\boldsymbol{D}_{\mathbb{Q}}\right)$ containing $R_{\Lambda}$. It follows from the isomorphism $C\left(\boldsymbol{L}_{\mathbb{Q}}\right) \cong \operatorname{End}_{W}\left(\boldsymbol{D}_{\mathbb{Q}}\right)$ of Proposition 2.4 that $R_{\Lambda}$ is a $W$-lattice in $\operatorname{End}_{W}\left(\boldsymbol{D}_{\mathbb{Q}}\right)$, and hence $\widetilde{R}_{\Lambda} / R_{\Lambda}$ is killed by some power of $p$, say $p^{M}$. Up to scaling by powers of $p$, there is a unique $W$-lattice $\widetilde{D} \subset \boldsymbol{D}_{\mathbb{Q}}$ such that $\widetilde{R}_{\Lambda} \widetilde{D}=\widetilde{D}$.

Now suppose $(G, \iota, \lambda, \varrho)$ is a $k$-point of $\mathscr{N}_{\Lambda}$. The quasi-isogeny $\varrho$ determines (up to scaling) a $W$-lattice $D \subset \boldsymbol{D}_{\mathbb{Q}}$ satisfying $R_{\Lambda} D=D$. It follows from $\widetilde{R}_{\Lambda} D=\widetilde{D}$ that

$$
p^{M} \widetilde{D} \subset D \subset \widetilde{D}
$$

after possibly rescaling $D$, and so there are integers $a<b$, independent of the point $(G, \iota, \lambda, \varrho)$, such that $p^{a} \boldsymbol{D} \subset D \subset p^{b} \boldsymbol{D}$. It follows from this bound and [Rapoport and Zink 1996, Corollary 2.29] that $\mathscr{N}_{\Lambda}$ is a closed subscheme of a projective scheme, hence is projective.

An obvious corollary of Proposition 2.19 is that every special lattice $L$ contains some $\Lambda^{\vee}$ (take $\left.\Lambda=\Lambda_{L}\right)$, and hence

$$
\mathscr{N}_{\text {red }}=\bigcup_{\Lambda} \mathscr{N}_{\Lambda}
$$


where the subscript red indicates the underlying reduced scheme. This union is not disjoint, as $\mathscr{N}_{\Lambda_{1}}^{ \pm} \subset \mathscr{N}_{\Lambda_{2}}^{ \pm}$whenever $\Lambda_{1} \subset \Lambda_{2}$. Define

$$
\mathscr{N}_{\Lambda}^{\circ}=\mathscr{N}_{\Lambda} \backslash \bigcup_{\Lambda^{\prime} \subsetneq \Lambda} \mathscr{N}_{\Lambda^{\prime}}
$$

so that (2-17) identifies

$$
\mathscr{N}_{\Lambda}^{\circ}(k)=\left\{\text { special lattices } L \text { such that } \Lambda_{L}=\Lambda\right\} .
$$

It follows easily that $\mathscr{N}_{\Lambda}=\biguplus_{\Lambda^{\prime} \subset \Lambda} \mathscr{N}_{\Lambda^{\prime}}^{\circ}$, and that

$$
\mathscr{N}_{\text {red }}=\biguplus_{\Lambda} \mathscr{N}_{\Lambda}^{\circ} \text {. }
$$

Abbreviate $\mathscr{N}_{\Lambda}^{ \pm}=\mathscr{N}_{\Lambda} \cap \mathscr{N}^{ \pm}$and $\mathscr{N}_{\Lambda}^{ \pm \circ}=\mathscr{N}_{\Lambda}^{\circ} \cap \mathscr{N}^{ \pm}$. By analogy with [Rapoport et al. 2014; Vollaard 2010; Vollaard and Wedhorn 2011], we call the decomposition (2-19) the Bruhat-Tits stratification of $\mathscr{N}_{\text {red }}$. This terminology should be taken with a grain of salt: unlike the situation in those references, the strata in (2-19) are not in bijection with the vertices in the Bruhat-Tits building of the group $J^{\text {der }}$. See Sections 2.7 and 3.6 below.

Remark 2.21. One could also define an $E$-vertex lattice to be an $\mathcal{O}_{E}$-lattice $\Lambda_{E} \subset$ $\left(\bigwedge_{E}^{2} \boldsymbol{D}_{\mathbb{Q}}\right)^{\Phi}$ such that $p \Lambda_{E} \subset \Lambda_{E}^{\vee} \subset \Lambda_{E}$, where the dual lattice is taken with respect to $\langle\cdot, \cdot\rangle$. The rule $\Lambda \mapsto \mathcal{O}_{E} \cdot \Lambda$ establishes a bijection between vertex lattices and $E$-vertex lattices, with inverse $\Lambda_{E} \mapsto\left\{x \in \Lambda_{E}: x^{\star}=x\right\}$. The action (2-11) of $\operatorname{GU}\left(\boldsymbol{D}_{\mathbb{Q}}\right)$ on $\bigwedge_{E}^{2} \boldsymbol{D}_{\mathbb{Q}}$ restricts to an action of $J$ on $\left(\bigwedge_{E}^{2} \boldsymbol{D}_{\mathbb{Q}}\right)^{\Phi}$, and induces an action of $J$ on the set of all $E$-vertex lattices. In particular, $J$ acts on the set of all vertex lattices. This action is compatible with the action of $J$ on $\mathscr{N}$ defined in Section 2.1, in the obvious sense: $g \mathscr{N}_{\Lambda}=\mathscr{N}_{g} \bullet \Lambda$. The restriction of this action to the subgroup $J^{0}$ of Remark 2.8 factors through the surjection $J^{0} \rightarrow \operatorname{SO}\left(L_{\mathbb{Q}}^{\Phi}\right)$, and agrees with the obvious action of $\operatorname{SO}\left(\boldsymbol{L}_{\mathbb{Q}}^{\Phi}\right)$ on the set of vertex lattices.

2.7. The Bruhat-Tits building. In [Garrett 1997, § 20.3] one finds a description of the Bruhat-Tits building of $\operatorname{SO}\left(\boldsymbol{L}_{\mathbb{Q}}^{\Phi}\right)$ in terms of lattices. See also [Tits 1979, $\S 1.16]$. We will translate this description into the language of our vertex lattices. Consider the set $\mathcal{V}^{\text {adm }}$ of all vertex lattices $\Lambda$ of type 2 or 6 . We call such vertex lattices admissible, and define an adjacency relation $\sim$ in $\mathcal{V}^{\mathrm{adm}}$ as follows: distinct admissible vertex lattices are adjacent $\left(\Lambda \sim \Lambda^{\prime}\right)$ if either:

(1) $\Lambda^{\prime} \subset \Lambda$ or $\Lambda^{\prime} \subset \Lambda$.

(2) $\Lambda$ and $\Lambda^{\prime}$ are both type- 6 and

$$
\begin{aligned}
& \operatorname{dim}_{\mathbb{F}_{p}}\left(\Lambda / \Lambda \cap \Lambda^{\prime}\right)=\operatorname{dim}_{\mathbb{F}_{p}}\left(\Lambda^{\prime} / \Lambda \cap \Lambda^{\prime}\right)=1, \\
& \operatorname{dim}_{\mathbb{F}_{p}}\left(\Lambda+\Lambda^{\prime} / \Lambda\right)=\operatorname{dim}_{\mathbb{F}_{p}}\left(\Lambda+\Lambda^{\prime} / \Lambda^{\prime}\right)=1 .
\end{aligned}
$$


If $\Lambda$ and $\Lambda^{\prime}$ are of type 6 and are adjacent, then $\Lambda \cap \Lambda^{\prime}$ is a vertex lattice of type 4 (so is not admissible). We construct an abstract simplicial complex with set of vertices $\mathcal{V}^{\text {adm }}$ as follows: An $m$-simplex $(0 \leq m \leq 2)$ of $\mathcal{V}^{\text {adm }}$ is a subset of $m+1$ admissible vertex lattices $\Lambda_{0}, \Lambda_{1}, \ldots, \Lambda_{m}$ which are mutually adjacent. The group $\operatorname{SO}\left(\boldsymbol{L}_{\mathbb{Q}}^{\Phi}\right)$ acts simplicially on $\mathcal{V}^{\text {adm }}$ by $g \in \operatorname{SO}\left(\boldsymbol{L}_{\mathbb{Q}}^{\Phi}\right)$ taking $\Lambda$ to $g \cdot \Lambda$.

Now consider the Bruhat-Tits building $\mathcal{B T}$ of $\operatorname{SO}\left(\boldsymbol{L}_{\mathbb{Q}}^{\Phi}\right)$. We will use the same symbol $\mathcal{B} \mathcal{T}$ to denote the underlying simplicial complex.

Proposition 2.22. There is an $\mathrm{SO}\left(\boldsymbol{L}_{\mathbb{Q}}^{\Phi}\right)$-equivariant simplicial bijection $\mathcal{B} \mathcal{T} \cong \mathcal{V}^{\mathrm{adm}}$. Furthermore, every vertex lattice of type 4 is contained in precisely two vertex lattices of type 6, and is equal to their intersection.

Proof. Define a new quadratic space $\left(V_{0}, Q_{0}\right)=\left(\boldsymbol{L}_{\mathbb{Q}}^{\Phi}, p^{-1} Q\right)$, and note that, by Proposition 2.6, $V_{0} \cong \mathbb{W}^{2} \oplus \mathbb{Q}_{p^{2}}$. The rule $\Lambda \mapsto p \Lambda$ defines a bijection from the set of vertex lattices in $L_{\mathbb{Q}}^{\Phi}$ to the set of lattices $L \subset V_{0}$ satisfying

$$
L \subset L^{*} \subset p^{-1} L
$$

Here $L^{*}$ is the dual lattice of $L$ with respect to the quadratic form $Q_{0}$. The isomorphism $\mathcal{B T} \cong \mathcal{V}^{\text {adm }}$ now follows from the description and properties of the affine building of $\operatorname{SO}\left(L_{\mathbb{Q}}^{\Phi}\right) \cong \operatorname{SO}\left(V_{0}\right)$ found in [Garrett 1997, Section 20.3].

If $\Lambda$ is a type- 4 vertex lattice, the lattice $L=p \Lambda$ in $V_{0}$ satisfies $\operatorname{dim}\left(L^{*} / L\right)=2$. Moreover, the $k$-quadratic space $L^{*} / L$ is a hyperbolic plane (choose a basis of $L$ for which the bilinear form has diagonal matrix, and use the fact that $V_{0}$ has Hasse invariant 1), and so contains exactly two isotropic lines. Those lines have the form $L_{1} / L$ and $L_{2} / L$, and $p^{-1} L_{1}$ and $p^{-1} L_{2}$ are the unique type-6 vertex lattices containing $\Lambda$.

We can also construct a simplicial complex $\mathcal{V}$ with vertices the set of all vertex lattices as follows (compare to [Rapoport et al. 2014, §3]). We call two distinct vertex lattices $\Lambda$ and $\Lambda^{\prime}$ neighbors if $\Lambda \subset \Lambda^{\prime}$ or $\Lambda^{\prime} \subset \Lambda$. An $m$-simplex $(m \leq 2)$ in $\mathcal{V}$ is formed by vertex lattices $\Lambda_{0}, \Lambda_{1}, \ldots, \Lambda_{m}$ such that any two members of this set are neighbors. The vertex lattices of type 4 are in bijection with pairs of adjacent type- 6 vertex lattices. Hence a vertex lattice of type 4 corresponds to an edge in the Bruhat-Tits building between type- 6 vertex lattices. From basic properties of the Bruhat-Tits building, we deduce the following:

Corollary 2.23. The group $\mathrm{SO}\left(\boldsymbol{L}_{\mathbb{Q}}^{\Phi}\right)$ acts transitively on the set of vertex lattices of a given type, and any two vertex lattices are connected by a sequence of adjacent vertices in $\mathcal{V}$. In particular, the group $J$, and even the subgroup $J^{0}$, act transitively on the set of vertex lattices of a given type (under the action of Remark 2.21). 


\section{Deligne-Lusztig varieties and the Bruhat-Tits strata}

In this section we show that for any vertex lattice $\Lambda$, the varieties

$$
\mathscr{N}_{\Lambda}=\mathscr{N}_{\Lambda}^{+} \uplus \mathscr{N}_{\Lambda}^{-} \quad \text { and } \quad \mathscr{N}_{\Lambda}^{\circ}=\mathscr{N}_{\Lambda}^{\circ+} \uplus \mathscr{N}_{\Lambda}^{\circ-}
$$

of Section 2.6 can be identified with varieties over $k$ defined purely in terms of the linear algebra of the $k$-quadratic space $\Omega=\left(\Lambda / \Lambda^{\vee}\right) \otimes_{\mathbb{F}_{p}} k$.

3.1. Deligne-Lusztig varieties. Let us recall the general definition of DeligneLusztig varieties. Suppose that $G_{0}$ is a connected reductive group over the finite field $\mathbb{F}_{p}$, and set $G=G_{0} \otimes_{\mathbb{F}_{p}} k$. We will also use the symbol $G$ to denote the abstract group of $k$-valued points of $G_{0}$. Denote by $\Phi: G \rightarrow G$ the Frobenius morphism. By Lang's theorem, $G_{0}$ is quasi-split, and so we may choose a maximal torus $T \subset G$ and a Borel subgroup containing $T$, both defined over $\mathbb{F}_{p}$. The Weyl group $W$ that corresponds to the pair $(T, B)$ is acted upon by $\Phi$, and the group $W$ with its $\Phi$-action does not depend on our choices. In fact, in [Deligne and Lusztig 1976] a Weyl group $W$ with $\Phi$-action is defined as a projective limit over all choices of pairs $(T, B)$, without having to assume that these pairs are $\Phi$-stable.

Let $\Delta^{*}=\left\{\alpha_{1}, \ldots, \alpha_{n}\right\}$ be the set of simple roots corresponding to the pair $(T, B)$, and consider the corresponding simple reflections $s_{i}=s_{\alpha_{i}}$ in the Weyl group $W$. For $I \subset \Delta^{*}$, let $W_{I}$ be the subgroup of $W$ generated by $\left\{s_{i}: i \in I\right\}$, and consider the corresponding parabolic subgroup $P_{I}=B W_{I} B$. The quotient $G / P_{I}$ parametrizes parabolic subgroups of $G$ of type $I$. Suppose $J \subset \Delta^{*}$ is another subset with corresponding standard parabolic $P_{J}$. Since

$$
G=\biguplus_{w \in W_{I} \backslash W / W_{J}} P_{I} w P_{J},
$$

we have a bijection

$$
P_{I} \backslash G / P_{J} \cong W_{I} \backslash W / W_{J} .
$$

Composing this with $G / P_{I} \times G / P_{J} \rightarrow P_{I} \backslash G / P_{J}$ given by $\left(g_{1}, g_{2}\right) \mapsto g_{1}^{-1} g_{2}$ defines the relative position invariant

$$
\text { inv : } G / P_{I} \times G / P_{J} \rightarrow W_{I} \backslash W / W_{J} .
$$

The Frobenius $\Phi: G \rightarrow G$ induces $\Phi: G / P_{I} \rightarrow G / P_{\Phi(I)}$.

Definition 3.1. For $w \in W_{I} \backslash W / W_{\Phi(I)}$, the Deligne-Lusztig variety $X_{P_{I}}(w)$ is the locally closed reduced subscheme of $G / P_{I}$ with $k$-points

$$
X_{P_{I}}(w)=\left\{g P_{I} \in G / P_{I}: \operatorname{inv}(g, \Phi(g))=w\right\} .
$$

The variety $X_{P_{I}}(w)$ is actually defined over the unique extension of degree $r$ of $\mathbb{F}_{p}$ in $k$, where $r$ is the smallest positive integer for which $\Phi^{r}(I)=I$. 
Proposition 3.2. The Deligne-Lusztig variety $X_{P_{I}}(w)$ is smooth of pure dimension

$$
\operatorname{dim} X_{P_{I}}(w)=\ell(w)+\operatorname{dim}\left(G / P_{I \cap \Phi(I)}\right)-\operatorname{dim}\left(G / P_{I}\right) .
$$

If $I=\Phi(I)$ then $\operatorname{dim} X_{P_{I}}(w)=\ell_{I}(w)-\ell\left(w_{I}\right)$, where $w_{I}$ is the longest element in $W_{I}$ and $\ell_{I}(w)$ is the maximal length of an element in $W_{I} w W_{I}$. Taking $I=\varnothing$, the variety $X_{B}(w)$ is irreducible of dimension $\operatorname{dim} X_{B}(w)=\ell(w)$.

Proof. This is standard. See, e.g., [Vollaard and Wedhorn 2011, Section 3.4].

Remark 3.3. If $w=1$, then $X_{P_{I}}(1)$ can be identified with the intersection of the image of the closed immersion $G / P_{I \cap \Phi(I)} \hookrightarrow G / P_{I} \times G / P_{\Phi(I)}$ with the graph of the Frobenius $\Phi: G / P_{I} \rightarrow G / P_{\Phi(I)}$. In particular $X_{P_{I}}(1)$ is projective. If

$$
\bigcup_{r \geq 0} \Phi^{r}(I)=\Delta^{*}
$$

then $X_{P_{I}}(1)$ is also irreducible, by [Bonnafé and Rouquier 2006].

3.2. An even orthogonal group. We now consider the case that $G_{0}$ is a nonsplit special orthogonal group in an even number of variables. Let $\Omega_{0}$ be an $\mathbb{F}_{p}$-vector space of dimension $2 d$ equipped with a nondegenerate nonsplit quadratic form. There is a basis $\left\{e_{1}, \ldots, e_{d}, f_{1}, \ldots, f_{d}\right\}$ of $\Omega=\Omega_{0} \otimes_{\mathbb{F}_{p}} k$ such that $\left\langle e_{1}, \ldots, e_{d}\right\rangle$ and $\left\langle f_{1}, \ldots, f_{d}\right\rangle$ are isotropic, $\left[e_{i}, f_{j}\right]=\delta_{i j}$, and the Frobenius

$$
\Phi=\mathrm{id} \otimes \sigma
$$

acting on $\Omega$ fixes $e_{i}$ and $f_{i}$ for $1 \leq i \leq d-1$, and interchanges $e_{d}$ with $f_{d}$. Note that $\Omega$ contains no $\Phi$-invariant Lagrangian subspaces. Abbreviate $G_{0}=\operatorname{SO}\left(\Omega_{0}\right)$ and $G=\mathrm{SO}(\Omega)$.

Denote by $\operatorname{OGr}(r)$ the scheme whose functor of points assigns to a $k$-scheme $S$ the set of all totally isotropic local $\mathcal{O}_{S}$-module direct summands $\mathcal{L} \subset \Omega \otimes_{k} \mathcal{O}_{S}$ of rank $r$. In particular, $\operatorname{OGr}(d)$ is the moduli space of Lagrangian subspaces of $\Omega$. Denote by $\operatorname{OGr}(d-1, d)$ the scheme whose functor of points assigns to a $k$ scheme $S$ the set of all flags of totally isotropic local $\mathcal{O}_{S}$-module direct summands $\mathcal{L}_{d-1} \subset \mathcal{L}_{d} \subset \Omega \otimes_{k} \mathcal{O}_{S}$ of rank $d-1$ and $d$, respectively. The following lemma is elementary:

Lemma 3.4. For each totally isotropic local $\mathcal{O}_{S}$-module direct summand

$$
\mathcal{L}_{d-1} \subset \Omega \otimes_{k} \mathcal{O}_{S}
$$

of rank $d-1$, there are exactly two totally isotropic local $\mathcal{O}_{S}$-module direct summands of rank $d$ containing $\mathcal{L}_{d-1}$.

In other words, the forgetful map $\operatorname{OGr}(d-1, d) \rightarrow \operatorname{OGr}(d-1)$ is a two-to-one cover. In fact, the Grassmannian $\operatorname{OGr}(d-1, d)$ has two connected components, 
which are interchanged by the action of any orthogonal transformation of determinant -1 . Each of the two components maps isomorphically to $\operatorname{OGr}(d-1)$ under the forgetful map. Label the two components as

$$
\operatorname{OGr}(d-1, d)=\mathrm{OGr}^{+}(d-1, d) \uplus \mathrm{OGr}^{-}(d-1, d)
$$

in such a way that the flags

$$
\left\langle e_{1}, \ldots, e_{d-1}\right\rangle \subset\left\langle e_{1}, \ldots, e_{d-1}, e_{d}\right\rangle
$$

and

$$
\left\langle e_{1}, \ldots, e_{d-1}\right\rangle \subset\left\langle e_{1}, \ldots, e_{d-1}, f_{d}\right\rangle
$$

define $k$-points of $\mathrm{OGr}^{+}(d-1, d)$ and $\mathrm{OGr}^{-}(d-1, d)$, respectively.

Denote by $\mathscr{X} \subset \operatorname{OGr}(d)$ the reduced closed subscheme with $k$-points

$$
\mathscr{X}=\{\mathcal{L} \in \operatorname{OGr}(d): \operatorname{dim}(\mathcal{L}+\Phi(\mathcal{L}))=d+1\} .
$$

There is a closed immersion $\mathscr{X} \rightarrow \operatorname{OGr}(d-1, d)$ sending

$$
\mathcal{L} \mapsto \mathcal{L} \cap \Phi(\mathcal{L}) \subset \mathcal{L}
$$

and the open and closed subvariety $\mathscr{X}^{ \pm}=\mathscr{X} \cap \mathrm{OGr}^{ \pm}(d-1, d)$ of $\mathscr{X}$ is identified with

$$
\mathscr{X}^{ \pm}=\left\{\mathcal{L}_{d-1} \subset \mathcal{L}_{d} \in \mathrm{OGr}^{ \pm}(d-1, d): \mathcal{L}_{d-1} \subset \Phi\left(\mathcal{L}_{d}\right)\right\} .
$$

Remark 3.5. Although we have defined $\operatorname{OGr}(d-1, d)$ and $\mathscr{X}$ as $k$-schemes, they both have natural $\mathbb{F}_{p}$-structures. The Frobenius morphism from $\operatorname{OGr}(d-1, d)$ to itself interchanges the flags (3-1) and (3-2), and hence interchanges the two connected components. It follows that $\mathscr{X}^{ \pm} \cong \sigma^{*} \mathscr{X}^{\mp}$, and that the individual

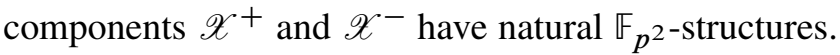

Our choice of basis of $\Omega$ determines a maximal $\Phi$-stable torus $T \subset G$. Set

$$
\begin{aligned}
& \mathcal{F}_{i}^{ \pm}=\left\langle e_{1}, \ldots, e_{i}\right\rangle \text { for } 1 \leq i \leq d-1, \\
& \mathcal{F}_{d}^{+}=\left\langle e_{1}, \ldots, e_{d-1}, e_{d}\right\rangle, \\
& \mathcal{F}_{d}^{-}=\left\langle e_{1}, \ldots, e_{d-1}, f_{d}\right\rangle .
\end{aligned}
$$

This gives two "standard" isotropic flags $\mathcal{F}_{\bullet}^{+}$and $\mathcal{F}_{\bullet}^{-}$in $\Omega$ satisfying $\mathcal{F}_{\bullet}^{ \pm}=\Phi\left(\mathcal{F}_{\bullet}^{\mp}\right)$. These flags have the same stabilizer $B \subset G$, which is a $\Phi$-stable Borel subgroup containing $T$. The corresponding set of simple reflections in the Weyl group is

$$
\left\{s_{1}, \ldots, s_{d-2}, t^{+}, t^{-}\right\}
$$

where:

- $s_{i}$ interchanges $e_{i}$ with $e_{i+1}, f_{i}$ with $f_{i+1}$, and fixes the other basis elements. 
- $t^{+}$interchanges $e_{d-1}$ with $e_{d}, f_{d-1}$ with $f_{d}$, and fixes the other basis elements.

- $t^{-}$interchanges $e_{d-1}$ with $f_{d}, f_{d-1}$ with $e_{d}$, and fixes the other basis elements. Notice that $\Phi\left(s_{i}\right)=s_{i}$ and $\Phi\left(t^{ \pm}\right)=t^{\mp}$, and so the products

$$
w^{ \pm}=t^{\mp} s_{d-2} \cdots s_{2} s_{1}
$$

are Coxeter elements: products of exactly one representative from each $\Phi$-orbit in the set of simple reflections above. More generally, define $w_{0}=1, w_{1}^{ \pm}=t^{\mp}$, and

$$
w_{r}^{ \pm}=t^{\mp} \cdot s_{d-2} \cdots s_{d-r}
$$

for $2 \leq r \leq d-1$. In particular, $w_{d-1}^{ \pm}=w^{ \pm}$. Define parabolic subgroups

$$
B=P_{d-1} \subset P_{d-2} \subset \cdots \subset P_{0} \subset P^{ \pm}
$$

of $G$ as follows: set $P_{d-1}=P_{d-2}=B$, and for $0 \leq r \leq d-2$ let $P_{r}$ be the parabolic corresponding to the set $\left\{s_{1}, \ldots, s_{d-(r+2)}\right\}$. Define $P^{ \pm}$to be the maximal parabolic corresponding to $\left\{s_{1}, \ldots, s_{d-2}, t^{ \pm}\right\}$. One can easily check that $P_{0}$ is the stabilizer in $G$ of $\mathcal{F}_{d-1}^{ \pm}$, and so

$$
G / P_{0} \cong \operatorname{OGr}(d-1) .
$$

More generally, $P_{r}$ is the stabilizer of the standard isotropic flag $\mathcal{F}_{d-r-1}^{ \pm} \subset \cdots \subset \mathcal{F}_{d}^{ \pm}$. Similarly, $P^{ \pm}$is the stabilizer of the Lagrangian subspace $\mathcal{F}_{d}^{ \pm}$, and so

$$
G / P^{ \pm} \cong \operatorname{OGr}(d) \text {. }
$$

Proposition 3.6. The isomorphism (3-6) identifies $\mathscr{X}^{ \pm}$with the Deligne-Lusztig variety $X_{P^{ \pm}}(1)$. In particular, $\mathscr{X}^{ \pm}$is projective, irreducible, and smooth of dimension $d-1$.

Proof. Note that $P_{0}=P^{+} \cap P^{-}$, and that the Frobenius $\Phi$ interchanges $P^{+}$ and $P^{-}$. The two projections $G / P_{0} \rightarrow G / P^{ \pm}$combine to give closed immersions

$$
i^{+}: G / P_{0} \rightarrow G / P^{+} \times G / P^{-} \text {and } i^{-}: G / P_{0} \rightarrow G / P^{-} \times G / P^{+},
$$

while the Frobenius induces morphisms $\Phi: G / P^{+} \rightarrow G / P^{-}$and $\Phi: G / P^{-} \rightarrow$ $G / P^{+}$with graphs $\Gamma_{\Phi}^{+} \subset G / P^{+} \times G / P^{-}$and $\Gamma_{\Phi}^{-} \subset G / P^{-} \times G / P^{+}$, respectively. The isomorphisms (3-5) and (3-6) identify the intersection of $\Gamma_{\Phi}^{ \pm}$and the image of $i^{ \pm}$with the set of flags $\mathcal{L}_{d-1} \subset \mathcal{L}_{d} \in \mathrm{OGr}^{ \pm}(d-1, d)$ such that $\mathcal{L}_{d-1} \subset \Phi\left(\mathcal{L}_{d}\right)$. By (3-3), this intersection is isomorphic to $\mathscr{X}^{+}$. All of the claims now follow from Remark 3.3, together with the dimension formula

$$
\operatorname{dim} X_{P^{ \pm}}(1)=\operatorname{dim}\left(G / P_{0}\right)-\operatorname{dim}\left(G / P^{ \pm}\right)=d-1
$$

of Proposition 3.2. 
It is also useful to view $\mathscr{X}^{ \pm}$as the closure of a Deligne-Lusztig variety in the flag variety $G / P_{0}$.

Lemma 3.7. The isomorphism $\mathrm{OGr}^{ \pm}(d-1, d) \cong \operatorname{OGr}(d-1) \cong G / P_{0}$ identifies $\mathscr{X}^{ \pm}$with the closure in $G / P_{0}$ of the Deligne-Lusztig variety

$$
X_{P_{0}}\left(t^{\mp}\right)=\left\{g \in G / P_{0}: \operatorname{inv}(g, \Phi(g))=t^{\mp}\right\} .
$$

Proof. Using (3-3), we may characterize the $k$-points of $\mathscr{X}^{ \pm}$by

$$
\begin{aligned}
\mathscr{X}^{ \pm}=\left\{\mathcal{L}_{d-1} \subset \mathcal{L}_{d} \in \mathrm{Gr}^{ \pm}(d-1, d)\right. & : \\
\Phi\left(\mathcal{L}_{d-1}\right) & \left.=\mathcal{L}_{d-1} \text { or } \mathcal{L}_{d-1}+\Phi\left(\mathcal{L}_{d-1}\right)=\Phi\left(\mathcal{L}_{d}\right)\right\} .
\end{aligned}
$$

Recalling the standard isotropic flags of (3-4), the rule $g \mapsto g \mathcal{F}_{d-1}^{ \pm} \subset g \mathcal{F}_{d}^{ \pm}$defines an isomorphism

$$
X_{P_{0}}(1) \cong\left\{\mathcal{L}_{d-1} \subset \mathcal{L}_{d} \in \operatorname{Gr}^{ \pm}(d-1, d): \mathcal{L}_{d-1}=\Phi\left(\mathcal{L}_{d-1}\right)\right\},
$$

while the same rule defines an isomorphism

$$
X_{P_{0}}\left(t^{\mp}\right) \cong\left\{\mathcal{L}_{d-1} \subset \mathcal{L}_{d} \in \mathrm{Gr}^{ \pm}(d-1, d): \mathcal{L}_{d-1}+\Phi\left(\mathcal{L}_{d-1}\right)=\Phi\left(\mathcal{L}_{d}\right)\right\} .
$$

Thus $\mathscr{X}^{ \pm}$is the disjoint union of $X_{P_{0}}(1)$ and $X_{P_{0}}\left(t^{\mp}\right)$. Elementary properties of the Bruhat order (see Section 8.5 of [Springer 1998], for example) imply that

$$
\overline{X_{P_{0}}\left(t^{\mp}\right)}=X_{P_{0}}(1) \uplus X_{P_{0}}\left(t^{\mp}\right),
$$

completing the proof.

The following proof is essentially the same as [Rapoport et al. 2014, Proposition 5.5].

Proposition 3.8. There is a stratification

$$
\mathscr{X}^{ \pm}=\biguplus_{r=0}^{d-1} X_{P_{r}}\left(w_{r}^{ \pm}\right)
$$

of $\mathscr{X}^{ \pm}$into a disjoint union of locally closed subvarieties. The stratum $X_{P_{r}}\left(w_{r}^{ \pm}\right)$ is smooth of pure dimension $r$, and has closure

$$
\overline{X_{P_{r}}\left(w_{r}^{ \pm}\right)}=X_{P_{0}}\left(w_{0}^{ \pm}\right) \uplus \cdots \uplus X_{P_{r}}\left(w_{r}^{ \pm}\right) .
$$

The highest-dimensional stratum $X_{P_{d-1}}\left(w_{d-1}^{ \pm}\right)=X_{B}\left(w^{ \pm}\right)$is irreducible, open, and dense.

Proof. Suppose $\mathcal{L}$ is a $k$-point of $\mathscr{X}^{ \pm} \subset \operatorname{Gr}(d)$, and define

$$
\mathcal{L}^{(i)}=\mathcal{L} \cap \Phi(\mathcal{L}) \cap \cdots \cap \Phi^{i}(\mathcal{L}) .
$$


An inductive argument, using

$$
\mathcal{L}^{(i)} \cap \Phi\left(\mathcal{L}^{(i)}\right)=\mathcal{L}^{(i-1)} \cap \Phi\left(\mathcal{L}^{(i-1)}\right) \cap \Phi^{2}\left(\mathcal{L}^{(i-1)}\right),
$$

shows that $\mathcal{L}^{(i+1)}$ has codimension at most 1 in $\mathcal{L}^{i}$. Denote by $\mathscr{X}_{r}^{ \pm} \subset \mathscr{X}^{ \pm}$the reduced closed subscheme whose $k$-valued points are those $\mathcal{L}$ satisfying $\mathcal{L}^{(r+2)}=$ $\mathcal{L}^{(r+1)}$. The complement $\mathscr{X}_{r}^{ \pm} \backslash \mathscr{X}_{r-1}^{ \pm}$is the locally closed subvariety of $\mathscr{X}^{ \pm}$ consisting of those $\mathcal{L}$ for which

$$
\mathcal{L}^{(r+2)}=\mathcal{L}^{(r+1)} \subsetneq \cdots \subsetneq \mathcal{L}^{(1)} \subsetneq \mathcal{L}^{(0)}=\mathcal{L} .
$$

Recalling that the parabolic subgroup $P_{r}$ is the stabilizer of the standard isotropic flag $\mathcal{F}_{d-r-1}^{ \pm} \subset \cdots \subset \mathcal{F}_{d}^{ \pm}$of length $r+2$, we obtain a morphism $\mathscr{X}_{r}^{ \pm} \backslash \mathscr{X}_{r-1}^{ \pm} \rightarrow G / P_{r}$ by sending $\mathcal{L}$ to the flag (3-7). By similar reasoning as [Rapoport et al. 2014, Proposition 5.5], this defines an isomorphism $\mathscr{X}_{r}^{ \pm} \backslash \mathscr{X}_{r-1}^{ \pm} \cong X_{P_{r}}\left(w_{r}^{ \pm}\right)$with inverse $g \mapsto g \mathcal{F}_{d}^{ \pm}$. All claims now follow easily.

3.3. A few special cases. We continue to let $G_{0}=\mathrm{SO}\left(\Omega_{0}\right)$, where $\Omega_{0}$ is the nonsplit quadratic space over $\mathbb{F}_{p}$ of dimension $2 d, \Omega=\Omega_{0} \otimes k$, and $G=\operatorname{SO}(\Omega)$. In the applications we will only need to consider $d \leq 3$, and in these cases the structure of the $k$-variety $\mathscr{X}$ (with its $\mathbb{F}_{p^{2}}$-structure of Remark 3.5) can be made more explicit.

(a) First suppose $d=1$. In this case $\mathscr{X}^{ \pm}$is a single point, defined over $\mathbb{F}_{p^{2}}$.

(b) Now suppose $d=2$. In this case $P_{1}=P_{0}=B$, and the stratification of Proposition 3.8 is

$$
\mathscr{X}^{ \pm}=X_{B}(1) \uplus X_{B}\left(t^{\mp}\right),
$$

where $X_{B}(1)$ is a 0 -dimensional closed subvariety and the open stratum $X_{B}\left(t^{\mp}\right)$ has dimension 1. The Dynkin diagram identity $D_{2}=A_{1} \times A_{1}$ corresponds to an exceptional isomorphism $\operatorname{Spin}(\Omega) \cong \mathrm{SL}_{2} \times \mathrm{SL}_{2}$. Indeed, the even Clifford algebra $C_{0}\left(\Omega_{0}\right)$ is isomorphic to $M_{2}\left(\mathbb{F}_{p^{2}}\right)$, and hence $C_{0}(\Omega) \cong M_{2}(k) \times M_{2}(k)$. This isomorphism restricts to an isomorphism of algebraic groups

$$
\operatorname{GSpin}(\Omega) \cong\left\{(x, y) \in \mathrm{GL}_{2} \times \mathrm{GL}_{2}: \operatorname{det}(x)=\operatorname{det}(y)\right\}
$$

over $k$, which in turn determines an isomorphism of $k$-varieties $G / P_{0} \cong \mathbb{P}^{1} \times \mathbb{P}^{1}$ in such a way that the Frobenius morphism on the left corresponds to $(x, y) \mapsto$ $(\Phi(y), \Phi(x))$ on the right. The subvarieties $\mathscr{X}^{ \pm} \subset G / P_{0}$ are identified with

$$
\begin{aligned}
& \mathscr{X}^{+}=\left\{(\Phi(x), x): x \in \mathbb{P}^{1}\right\}, \\
& \mathscr{X}^{-}=\left\{(x, \Phi(x)): x \in \mathbb{P}^{1}\right\} .
\end{aligned}
$$

Therefore, both $\mathscr{X}^{+}$and $\mathscr{X}^{-}$are isomorphic (over $\mathbb{F}_{p^{2}}$ ) to $\mathbb{P}^{1}$. The closed stratum $X_{B}(1)$ corresponds to the $\mathbb{F}_{p^{2}}$-rational points of $\mathbb{P}^{1}$. 
(c) Finally, suppose $d=3$. In this case

$$
\mathscr{X}^{ \pm}=X_{P_{0}}(1) \uplus X_{B}\left(t^{\mp}\right) \uplus X_{B}\left(t^{\mp} s_{1}\right) .
$$

The open stratum $X_{B}\left(t^{\mp} s_{1}\right)$ has dimension 2, the stratum $X_{B}\left(t^{\mp}\right)$ is locally closed of dimension 1, and the closed stratum $X_{P_{0}}(1)$ has dimension 0 . To continue, we will use the Dynkin diagram isomorphism $D_{3}=A_{3}$, corresponding to an isomorphism between the adjoint forms of $G$ and a unitary group in 4 variables, as in Proposition 2.7 and Remark 2.8.

Let $\mathcal{V}_{0}$ be the 4-dimensional $\mathbb{F}_{p^{2}}$-vector space with basis $e_{1}, e_{2}, e_{3}, e_{4}$, endowed with the split $\mathbb{F}_{p^{2}} / \mathbb{F}_{p}$-Hermitian form defined by $\left\langle e_{i}, e_{5-j}\right\rangle=\delta_{i j}$. Denote by $U_{0}$ the unitary group of $\mathcal{V}_{0}$, an algebraic group over $\mathbb{F}_{p}$, so that $U=U_{0} \times_{\mathbb{F}_{p}} k$ acts on $\mathcal{V}=\mathcal{V}_{0} \otimes_{\mathbb{F}_{p}} k$. Recall the isomorphism $\mathbb{F}_{p^{2}} \otimes_{\mathbb{F}_{p}} k \simeq k \oplus k$ defined by $x \otimes a \mapsto$ $\left(x a, x^{p} a\right)$, and denote by $\epsilon_{0}$ and $\epsilon_{1}$ the idempotents that correspond to $(1,0)$ and $(0,1)$, so that

$$
\epsilon_{0} \mathcal{V} \cong \mathcal{V}_{0} \otimes_{\mathbb{F}_{p^{2}}} k
$$

The action of $U$ on $\epsilon_{0} \mathcal{V}$ defines an isomorphism $U \cong \mathrm{GL}_{4}$. The diagonal torus and the standard Borel subgroup of upper-triangular matrices in $\mathrm{GL}_{4}$ give a maximal torus and a Borel subgroup of $U$, both defined over $\mathbb{F}_{p}$. Given $r, s \geq 0$ with $r+s=4$, the pair $(r, s)$, viewed as an ordered partition of 4 , defines a parabolic subgroup $P_{(r, s)}$ containing $B$ with Levi component $\mathrm{GL}_{r} \times \mathrm{GL}_{s}$. The parabolic subgroup $P_{(r, s)}$ is defined over $\mathbb{F}_{p^{2}}$ and satisfies $\Phi\left(P_{(r, s)}\right)=P_{(s, r)}$.

Let $\operatorname{Gr}(r)$ be the Grassmanian of $r$-planes in $\epsilon_{0} \mathcal{V}$. The above isomorphism $U \cong \mathrm{GL}_{4}$ induces an isomorphism $U / P_{(r, s)} \cong \mathrm{Gr}(r)$ defined over $\mathbb{F}_{p^{2}}$, and the Frobenius morphism $\Phi: U / P_{(r, s)} \rightarrow U / P_{(s, r)}$ corresponds to a morphism

$$
\Phi: \operatorname{Gr}(r) \rightarrow \operatorname{Gr}(s)
$$

which can be described, as in [Vollaard 2010], as follows. Consider the $k$-valued form $\langle\langle\cdot, \cdot\rangle\rangle$ on (3-9) defined by

$$
\langle\langle x \otimes a, y \otimes b\rangle\rangle=\langle x, y\rangle \otimes a b^{p} .
$$

It is $k$-linear in the first variable but Frobenius-semilinear in the second. For a subspace $\mathcal{U} \subset \epsilon_{0} \mathcal{V}$, denote by $\mathcal{U}^{\llcorner}$the perpendicular of $\mathcal{U}$ for the form $\langle\langle\cdot, \cdot\rangle\rangle$. If $\mathcal{U}$ is $\mathbb{F}_{p^{2}}$-rational then $\mathcal{U}^{\llcorner}=\mathcal{U}^{\perp}$. If $\operatorname{dim}_{k}(\mathcal{U})=r$ then $\operatorname{dim}_{k}\left(\mathcal{U}^{\llcorner}\right)=s$, and, according to [Vollaard 2010, Lemma 2.12], the morphism (3-10) is given by $\Phi(\mathcal{U})=\mathcal{U}^{\llcorner}$on $k$-valued points. Using this description of $\Phi$, the unitary Deligne-Lusztig variety $X_{P_{(r, s)}}(1) \subset \operatorname{Gr}(r)$ is seen to be

$$
X_{P_{(r, s)}}(1)= \begin{cases}\left\{\mathcal{U} \subset \epsilon_{0} \mathcal{V}: \operatorname{dim}_{k}(\mathcal{U})=r, \mathcal{U} \subset \mathcal{U}^{\llcorner}\right\} & \text {if } r \leq s, \\ \left\{\mathcal{U} \subset \epsilon_{0} \mathcal{V}: \operatorname{dim}_{k}(\mathcal{U})=r, \mathcal{U}^{\llcorner} \subset \mathcal{U}\right\} & \text { if } s \leq r\end{cases}
$$


By Remark 3.3 these Deligne-Lusztig varieties are projective and smooth.

By inspecting the Dynkin diagram identity $D_{3}=A_{3}$ we can see that the exceptional isomorphism between the adjoint forms of $G$ and $U$ can be chosen so that the parabolic subgroups $P^{+}, P^{-}$and $P_{0}$ of $G$ correspond to $P_{(1,3)}, P_{(3,1)}$ and $P_{(1,3)} \cap P_{(3,1)}$ of $U \cong \mathrm{GL}_{4}$ respectively. Therefore, the Deligne-Lusztig variety $\mathscr{X}^{+}$is isomorphic to the unitary Deligne-Lusztig variety

$$
X_{P_{(1,3)}}(1)=\left\{\mathcal{U} \subset k^{4}: \operatorname{dim}_{k}(\mathcal{U})=1, \mathcal{U} \subset \mathcal{U}^{\llcorner}\right\} .
$$

Similarly $\mathscr{X}^{-}$is isomorphic to the unitary Deligne-Lusztig variety

$$
X_{P_{(3,1)}}(1)=\left\{\mathcal{U} \subset k^{4}: \operatorname{dim}_{k}(\mathcal{U})=3, \mathcal{U}^{\llcorner} \subset \mathcal{U}\right\} .
$$

Therefore, both $\mathscr{X}^{+}$and $\mathscr{X}^{-}$are isomorphic over $\mathbb{F}_{p^{2}}$ to the smooth hypersurface in $\mathbb{P}^{3}$ given by the homogeneous equation

$$
x_{1} x_{4}^{p}+x_{2} x_{3}^{p}+x_{3} x_{2}^{p}+x_{4} x_{1}^{p}=0 .
$$

In fact, since all nondegenerate Hermitian forms on $\mathcal{V}_{0}=\mathbb{F}_{p^{2}}^{4}$ are isomorphic we can also determine equations for the unitary Deligne-Lusztig varieties using the Hermitian form given by the identity matrix $I_{4}$. This gives the Fermat hypersurface

$$
x_{0}^{p+1}+x_{1}^{p+1}+x_{2}^{p+1}+x_{3}^{p+1}=0,
$$

which is isomorphic to the surface above.

The stratification (3-8) of $\mathscr{X}^{+}$now corresponds to the stratification of the unitary Deligne-Lusztig variety $X_{P_{(1,3)}}$ (1) studied in [Vollaard 2010, Theorem 2.15]. The Frobenius $\sigma: k \rightarrow k$ defines an operator on $\mathcal{V}$ which interchanges the two summands $\mathcal{V}=\epsilon_{0} \mathcal{V} \oplus \epsilon_{1} \mathcal{V}$. Thus we obtain an operator $\tau=\sigma^{2}$ on $\epsilon_{0} \mathcal{V}$. Any $k$-subspace $\mathcal{U} \subset \epsilon_{0} \mathcal{V}$ satisfies $\tau(\mathcal{U})=\left(\mathcal{U}^{\llcorner}\right)^{\llcorner}$. The open 2-dimensional stratum of $X_{P_{(1,3)}}(1)$ has $k$-valued points corresponding to lines $\mathcal{U}$ such that

$$
\begin{aligned}
\operatorname{dim}_{k}(\mathcal{U}+\tau(\mathcal{U})) & =2, \\
\operatorname{dim}_{k}\left(\mathcal{U}+\tau(\mathcal{U})+\tau^{2}(\mathcal{U})\right) & =3 .
\end{aligned}
$$

The 1-dimensional stratum has $k$-valued points corresponding to lines $\mathcal{U}$ such that

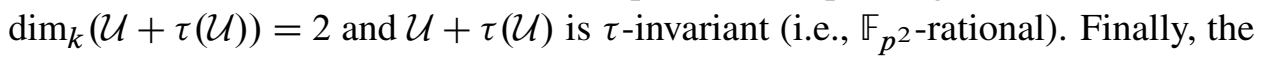
0 -dimensional stratum consists of $k$-valued points corresponding to lines $\mathcal{U}$ which are $\tau$-invariant. In other words, the 0 -dimensional stratum of $\mathscr{X}^{+}$is just the set of $\mathbb{F}_{p^{2}}$-rational points. For a $k$-valued point $\mathcal{U}$ on the 1 -dimensional stratum, set

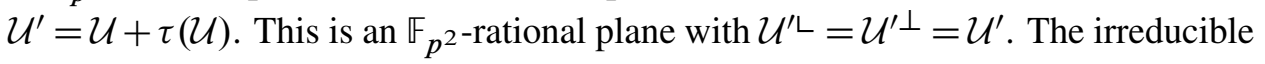
components of the 1-dimensional stratum are parametrized by such planes. Indeed, conversely, given an $\mathbb{F}_{p^{2}}$-rational plane $\mathcal{U}^{\prime}$ which is isotropic $\left(\mathcal{U}^{\prime}=\mathcal{U}^{\prime}\right)$, we obtain a closed subscheme of $X_{P_{(1,3)}}(1)$ with points corresponding to all lines $\mathcal{U}$ with 
$\mathcal{U} \subset \mathcal{U}^{\prime}$. This subscheme is isomorphic to $\mathbb{P}^{1}$ and gives the Zariski closure of the corresponding irreducible component of the 1-dimensional stratum.

We can now also determine the number of components of the strata:

- The 0 -dimensional stratum consists of $\left(p^{3}+1\right)\left(p^{2}+1\right)$ points. Indeed, observe that, as in [Vollaard and Wedhorn 2011, Example 5.6], we can calculate that

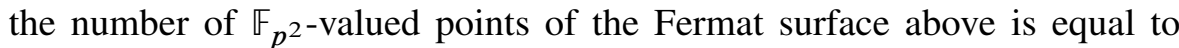
$\left(p^{3}+1\right)\left(p^{2}+1\right)$. (Note that there is a typographical error in [loc. cit.]: the summation for $\Sigma_{l}$ should start at $j=0$.) This is equal to the number of

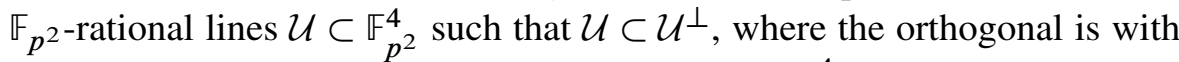
respect to the (standard) Hermitian form $\langle\cdot, \cdot\rangle$ on $\mathbb{F}_{p^{2}}^{4}$. Therefore, we have $\left(p^{3}+1\right)\left(p^{2}+1\right)$ components of the 0 -dimensional stratum.

- The 1-dimensional stratum has $\left(p^{3}+1\right)(p+1)$ components. Note that by the above, the Zariski closure of each component is isomorphic to a projective line $\mathbb{P}^{1}$ over $\mathbb{F}_{p^{2}}$ and the corresponding component is the complement of all $\mathbb{F}_{p^{2} \text {-rational points in this line. To determine the number of irreducible compo- }}$ nents of the 1-dimensional stratum, we start by counting the number of such

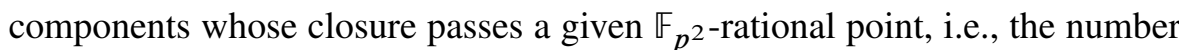
of copies of $\mathbb{P}^{1}$ in our configuration that cross at that point: By the above, this count is given by the number of $\mathbb{F}_{p^{2}}$-rational planes $\mathcal{U}^{\prime}$ which are isotropic and

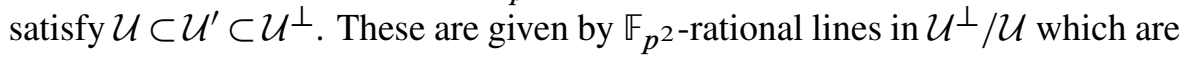
isotropic for the induced hermitian form. We can easily see that there are exactly $p+1$ such lines. Since there are a total of $\left(p^{3}+1\right)\left(p^{2}+1\right) \mathbb{F}_{p^{2}}$-rational points, each belonging on $p+1$ projective lines which each have $p^{2}+1$ points, we conclude that there are exactly $\left(p^{3}+1\right)(p+1)$ projective lines in our configuration. The same discussion applies to $\mathscr{X}^{-}$.

3.4. Deligne-Lusztig varieties and the Bruhat-Tits stratification. Now we relate the varieties $\mathscr{X}$ studied above to the varieties $\mathscr{N}_{\Lambda}^{\circ} \subset \mathscr{N}_{\Lambda}$ of Section 2.6. Fix a vertex lattice $\Lambda$ of type $2 d \in\{2,4,6\}$, and endow the $2 d$-dimensional $\mathbb{F}_{p}$-vector space

$$
\Omega_{0}=\Lambda / \Lambda^{\vee}
$$

with the nondegenerate $\mathbb{F}_{p}$-valued quadratic form $q(x)=p Q(x)$ induced by the quadratic form $Q$ on $\boldsymbol{L}_{\mathbb{Q}}^{\Phi}$. Set $\Omega=\Omega_{0} \otimes_{\mathbb{F}_{p}} k$, and let $G$ be the special orthogonal group of $\Omega$. Note that $\Omega_{0}$ is nonsplit: the existence of a $d$-dimensional totally isotropic subspace in $\Omega_{0}$ would imply the existence of a self-dual lattice in $\boldsymbol{L}_{\mathbb{Q}}^{\Phi}$, contradicting the Hasse invariant calculation of Proposition 2.6.

Recall from Section 3.2 the reduced closed subscheme $\mathscr{X}=\mathscr{X}_{\Lambda} \subset \operatorname{OGr}(d)$ whose $k$-points are the Lagrangian subspaces $\mathcal{L} \subset \Omega$ with

$$
\operatorname{dim}_{k}(\mathcal{L}+\Phi(\mathcal{L}))=d+1
$$


The Lagrangian subspaces of $\Omega$ are in bijection with the $W$-lattices $L \subset \boldsymbol{L}_{\mathbb{Q}}$ satisfying $L=L^{\vee}$ and $\Lambda^{\vee} \subset L$, and the condition (3-11) is equivalent to $L$ being a special lattice. Combining this with (2-18), we obtain bijections

$$
\mathscr{X}_{\Lambda}(k) \cong\left\{\text { special lattices } L \subset \boldsymbol{L}_{\mathbb{Q}} \text { such that } \Lambda^{\vee} \subset L\right\} \cong \mathscr{N}_{\Lambda}(k) \text {. }
$$

Theorem 3.9. There is an isomorphism of $k$-schemes $\mathscr{N}_{\Lambda} \cong \mathscr{X}_{\Lambda}$ inducing the above bijection on k-points. After possibly relabeling the two connected components of $\mathscr{X}_{\Lambda}=\mathscr{X}_{\Lambda}^{+} \uplus \mathscr{X}_{\Lambda}^{-}$, we may assume that this isomorphism identifies $\mathscr{N}_{\Lambda}^{ \pm} \cong \mathscr{X}_{\Lambda}^{ \pm}$.

Proof. Let $R$ be a reduced $k$-algebra of finite type. Given an $R$-valued point $(G, \iota, \lambda, \varrho) \in \mathscr{N}_{\Lambda}(R)$, there is an induced map of $\mathbb{Z}_{p}$-modules

$$
\Lambda^{\vee} \rightarrow \operatorname{End}(G)
$$

defined by $x \mapsto \varrho^{-1} \circ x \circ \varrho$. Let $\mathcal{D}$ be the covariant Grothendieck-Messing crystal of $G$, evaluated at the trivial divided power thickening $\operatorname{Spec}(R) \rightarrow \operatorname{Spec}(R)$, so that $\mathcal{D}$ is a locally free $R$-module sitting in an exact sequence

$$
0 \longrightarrow \mathcal{D}_{1} \longrightarrow \mathcal{D} \longrightarrow \operatorname{Lie}(G) \longrightarrow 0 .
$$

The formation of the pair $\mathcal{D}_{1} \subset \mathcal{D}$ is functorial in $G$, so there are induced morphisms of $R$-modules

$$
\psi:\left(\Lambda^{\vee} / p \Lambda^{\vee}\right) \otimes_{\mathbb{F}_{p}} R \rightarrow \operatorname{End}_{R}(\mathcal{D})
$$

and

$$
\psi_{1}:\left(\Lambda^{\vee} / p \Lambda^{\vee}\right) \otimes_{\mathbb{F}_{p}} R \rightarrow \operatorname{End}_{R}\left(\mathcal{D}_{1}\right)
$$

with $\operatorname{ker}(\psi) \subset \operatorname{ker}\left(\psi_{1}\right)$. Given $x \in \operatorname{ker}\left(\psi_{1}\right)$ and $y \in\left(\Lambda^{\vee} / p \Lambda^{\vee}\right) \otimes_{\mathbb{F}_{p}} R$, the endomorphism

$$
[x, y]=x \circ y+y \circ x \in \operatorname{End}_{R}\left(\mathcal{D}_{1}\right)
$$

is trivial. Thus the kernel of $\psi_{1}$ is contained in the radical of the quadratic space $\left(\Lambda^{\vee} / p \Lambda^{\vee}\right) \otimes_{\mathbb{F}_{p}} R$, which is $\left(p \Lambda / p \Lambda^{\vee}\right) \otimes_{\mathbb{F}_{p}} R$. Let $\mathcal{L}^{\sharp} \subset \mathcal{K}$ be the images of $\operatorname{ker}(\psi) \subset \operatorname{ker}\left(\psi_{1}\right)$ under the obvious isomorphism

$$
\left(p \Lambda / p \Lambda^{\vee}\right) \otimes_{\mathbb{F}_{p}} R \cong\left(\Lambda / \Lambda^{\vee}\right) \otimes_{\mathbb{F}_{p}} R \cong \Omega \otimes_{k} R .
$$

When $R=k$, the point $(G, \iota, \lambda, \varrho)$ corresponds to some Dieudonné lattice $D$ with $D_{1}=V D$, and $\mathcal{D}_{1} \subset \mathcal{D}$ is canonically identified with $D_{1} / p D \subset D / p D$. Under these identifications,

$$
\begin{aligned}
\operatorname{ker}(\psi) & =\left\{x \in\left(p \Lambda / p \Lambda^{\vee}\right) \otimes_{\mathbb{F}_{p}} k: x D \subset p D\right\}, \\
\operatorname{ker}\left(\psi_{1}\right) & =\left\{x \in\left(p \Lambda / p \Lambda^{\vee}\right) \otimes_{\mathbb{F}_{p}} k: x D_{1} \subset p D\right\},
\end{aligned}
$$

and so

$$
\begin{aligned}
\mathcal{L}^{\sharp} & =\left\{x \in\left(\Lambda / \Lambda^{\vee}\right) \otimes k: x D \subset D\right\}, \\
\mathcal{K} & =\left\{x \in\left(\Lambda / \Lambda^{\vee}\right) \otimes k: x D_{1} \subset D\right\} .
\end{aligned}
$$


If we identify a subspace of $\left(\Lambda / \Lambda^{\vee}\right) \otimes k$ with the lattice in $\boldsymbol{L}_{\mathbb{Q}}$ that it generates, then $\mathcal{L}^{\sharp}$ corresponds to the lattice $L^{\#}=\left\{x \in \boldsymbol{L}_{\mathbb{Q}}: x D \subset D\right\}$ of Theorem 2.12, and $\mathcal{K}$ corresponds to $L+L^{\sharp}=\left\{x \in \boldsymbol{L}_{\mathbb{Q}}: x D_{1} \subset D\right\}$. In particular, $\mathcal{L}^{\sharp}$ is totally isotropic of dimension $d$ and $\mathcal{K}$ has dimension $d+1$. Moreover, the quadratic space $\mathcal{K} / \mathcal{K}^{\perp}$ is a hyperbolic plane, and so has precisely two isotropic lines. One of them is $\mathcal{L}^{\sharp}$, and the other is the subspace $\mathcal{L}$ corresponding to $L=\left\{x \in \boldsymbol{L}_{\mathbb{Q}}: x D_{1} \subset D_{1}\right\}$.

For a general reduced $R$ of finite type, it follows from the previous paragraph (use Exercise X.16 of [Lang 2002] and the fact that $R$ is a Jacobson ring) that $\mathcal{L}^{\sharp}$ is a totally isotropic rank- $d$ local direct summand of $\Omega \otimes_{k} R$ and $\mathcal{K}$ is a rank- $(d+1)$ local direct summand. By Lemma 3.4 there is a unique totally isotropic rank- $d$ local direct summand $\mathcal{L} \neq \mathcal{L}^{\#}$ of $\Omega \otimes_{k} R$ contained in $\mathcal{K}$. As $\mathscr{N}_{\Lambda}$ is itself reduced and locally of finite type, the construction $(G, \iota, \lambda, \varrho) \mapsto \mathcal{L}$ defines a morphism of $k$-schemes

$$
\mathscr{N}_{\Lambda} \rightarrow \operatorname{OGr}(d)
$$

inducing the desired bijection $\mathscr{N}_{\Lambda}(k) \cong \mathscr{X}_{\Lambda}(k)$ on $k$-valued points. As the arguments of Section 2.4 were all done over an arbitrary extension of $k$, the above morphism induces a bijection $\mathscr{N}_{\Lambda}\left(k^{\prime}\right) \cong \mathscr{X}_{\Lambda}\left(k^{\prime}\right)$ for every field extension $k^{\prime} / k$. The morphism $\mathscr{N}_{\Lambda} \rightarrow \mathscr{X}_{\Lambda}$ is therefore birational, quasi-finite, and proper (by Proposition 2.20). As $\mathscr{X}_{\Lambda}$ is smooth (and therefore normal), Zariski's main theorem implies $\mathscr{N}_{\Lambda} \cong \mathscr{X}_{\Lambda}$. The claim about connected components is obvious.

3.5. The main results. Now we state our main results about the structure of the underlying reduced subscheme $\mathscr{N}_{\text {red }}=\mathscr{N}_{\text {red }}^{+} \sqcup \mathscr{N}_{\text {red }}^{-}$of $\mathscr{N}$. Recall from Section 2.6 that $\mathscr{N}_{\text {red }}^{ \pm}$is covered by the closed subschemes $\mathscr{N}_{\Lambda}^{ \pm}$as $\Lambda$ runs over the vertex lattices of type $t_{\Lambda}=2 d_{\Lambda} \in\{2,4,6\}$ in the 6-dimensional $\mathbb{Q}_{p}$-quadratic space $\boldsymbol{L}_{\mathbb{Q}}^{\Phi}$, and that their intersections are given by the simple rule

$$
\mathscr{N}_{\Lambda_{1}}^{ \pm} \cap \mathscr{N}_{\Lambda_{2}}^{ \pm}= \begin{cases}\mathscr{N}_{\Lambda_{1} \cap \Lambda_{2}}^{ \pm} & \text {if } \Lambda_{1} \cap \Lambda_{2} \text { is a vertex lattice, } \\ \varnothing & \text { otherwise }\end{cases}
$$

where, as before, the left-hand side is understood to mean the reduced scheme underlying the scheme-theoretic intersection. In other words, the combinatorics of the intersections are controlled by the combinatorics of the simplicial complex $\mathcal{V}$ of Section 2.7.

Theorem 3.10. The $k$-variety $\mathscr{N}_{\Lambda}^{ \pm}$is projective, smooth, and irreducible of dimension $d_{\Lambda}-1$. Moreover:

(1) If $d_{\Lambda}=1$, then $\mathscr{N}_{\Lambda}^{ \pm}$is a single point.

(2) If $d_{\Lambda}=2$, then $\mathscr{N}_{\Lambda}^{ \pm}$is isomorphic to $\mathbb{P}^{1}$.

(3) If $d_{\Lambda}=3$, then $\mathscr{N}_{\Lambda}^{ \pm}$is isomorphic to the Fermat hypersurface

$$
x_{0}^{p+1}+x_{1}^{p+1}+x_{2}^{p+1}+x_{3}^{p+1}=0 .
$$


Proof. Combine Theorem 3.9 with the discussion of Section 3.3.

Theorem 3.11. Under the isomorphism $\mathscr{X}_{\Lambda}^{ \pm} \cong \mathscr{N}_{\Lambda}^{ \pm}$, the stratification of Proposition 3.8 and the stratification

$$
\mathscr{N}_{\Lambda}^{ \pm}=\biguplus_{\Lambda^{\prime} \subset \Lambda} \mathscr{N}_{\Lambda^{\prime}}^{ \pm \circ}
$$

of Section 2.6 are related by

$$
X_{P_{r}}\left(w_{r}^{ \pm}\right) \cong \biguplus_{\substack{\Lambda^{\prime} \subset \Lambda \\ d_{\Lambda^{\prime}}=r+1}} \mathscr{N}_{\Lambda^{\prime}}^{ \pm \circ}
$$

for all $0 \leq r \leq d_{\Lambda}-1$. In particular (by taking $r=d_{\Lambda}-1$ ), the dense open subvariety $\mathscr{N}_{\Lambda}^{ \pm \circ}$ is isomorphic to the Deligne-Lusztig variety $X_{B}\left(w^{ \pm}\right)$associated with a Coxeter element.

Proof. For each special lattice $L$, we defined in Proposition 2.19 a sequence of lattices

$$
L=L^{(0)} \subsetneq L^{(1)} \subsetneq \cdots \subsetneq L^{(d)}=L^{(d+1)}
$$

by $L^{(r)}=L+\Phi(L)+\cdots+\Phi^{r}(L)$, and a type- $2 d$ vertex lattice

$$
\Lambda_{L}=\left\{x \in L^{(d)}: \Phi(x)=x\right\} .
$$

The bijection (2-18) identifies $\mathscr{N}_{\Lambda}^{ \pm}(k)$ with the set of special lattices $L$ with $\Lambda_{L} \subset \Lambda$, and the $k$-points of the right-hand side of (3-12) correspond to those $L$ for which $\Lambda_{L}$ has type $2 r+2$; in other words, those $L$ for which

$$
L=L^{(0)} \subsetneq L^{(1)} \subsetneq \cdots \subsetneq L^{(r+1)}=L^{(r+2)} .
$$

If we instead define $\underline{L}^{(r)}=L \cap \Phi(L) \cap \cdots \cap \Phi^{r}(L)$, this condition is equivalent to

$$
\underline{L}^{(r+2)}=\underline{L}^{(r+1)} \subsetneq \cdots \subsetneq \underline{L}^{(1)} \subsetneq \underline{L}^{(0)}=L .
$$

In the proof of Proposition 3.8, this is the same as the condition defining the strata $X_{P_{r}}\left(w_{r}^{ \pm}\right)$.

Theorem 3.12. The reduced $k$-scheme $\mathscr{N}_{\text {red }}$ is equidimensional of dimension two. It has two connected components, $\mathscr{N}_{\text {red }}^{+}$and $\mathscr{N}_{\text {red }}^{-}$, and these connected components are isomorphic. The irreducible components of $\mathscr{N}_{\text {red }}$ are precisely the closed subschemes $\mathscr{N}_{\Lambda}^{ \pm}$as $\Lambda$ varies over the type- 6 vertex lattices. Furthermore:

(1) For each irreducible component $\mathscr{N}_{\Lambda}$, there are exactly $\left(p^{3}+1\right)(p+1)$ irreducible components $\mathscr{N}_{\Lambda^{\prime}}$ such that $\mathscr{N}_{\Lambda} \cap \mathscr{N}_{\Lambda^{\prime}} \cong \mathbb{P}^{1}$, and $\left(p^{3}+1\right)\left(p^{2}+1\right)$ irreducible components $\mathscr{N}_{\Lambda^{\prime}}$ such that $\mathscr{N}_{\Lambda} \cap \mathscr{N}_{\Lambda^{\prime}}$ consists of a single point.

(2) For each type-4 vertex lattice $\Lambda$, the closed subscheme $\mathscr{N}_{\Lambda} \cong \mathbb{P}^{1}$ is contained in exactly two irreducible components, and is equal to their intersection. 
Proof. The isomorphism $\mathscr{N}_{\text {red }}^{+} \cong \mathscr{N}_{\text {red }}^{-}$follows from the isomorphism $\mathscr{N}^{+} \cong \mathscr{N}^{-}$of Section 2.1. The connectedness of $\mathscr{N}_{\text {red }}^{ \pm}$follows from Corollary 2.23. The remaining claims are clear from the theorems above and the discussion of Section 3.3.

3.6. Hermitian vertex lattices. As in [Rapoport et al. 2014; Vollaard 2010; Vollaard and Wedhorn 2011], it is possible to describe the stratification of $\mathscr{N}$ in terms of the Bruhat-Tits building of the special unitary group $J^{\text {der }}$, although in our setting the description in these terms is slightly convoluted. Recall from Remark 2.8 the central isogeny $J^{\text {der }} \rightarrow \operatorname{SO}\left(L_{\mathbb{Q}}^{\Phi}\right)$. Using [Bruhat and Tits $1984, \S 4.2 .15$ ], we see that this gives an identification of the building $\mathcal{B T}$ of $\operatorname{SO}\left(\boldsymbol{L}_{\mathbb{Q}}^{\Phi}\right)$, which was described in Section 2.7, with the building of $J^{\text {der }}$. Therefore, using [Vollaard 2010] and $J^{\text {der }} \cong \mathrm{SU}(T)$, we can see that the underlying simplicial complex of the building $\mathcal{B} \mathcal{T}$ can also be described using $\mathcal{O}_{E}$-lattices in the split Hermitian space $T$ of dimension 4 over $E$.

We say that an $\mathcal{O}_{E}$-lattice $\Xi \subset T$ is a Hermitian vertex lattice if

$$
\Xi \subset \Xi^{\vee} \subset p^{-1} \Xi .
$$

The type of $\Xi$ is $\operatorname{dim}_{\mathbb{F}_{p^{2}}}\left(\Xi^{\vee} / \Xi\right)$; the type can be 0,2 or 4 . As in [Vollaard 2010], these Hermitian vertex lattices correspond bijectively to the vertices of the BruhatTits building of $\mathrm{SU}(T)$. The action of the group $\mathrm{SU}(T)$ preserves the vertex type and is transitive on the set of vertices of a given type. The simplicial structure of the building of $\mathrm{SU}(T)$ is generated, as above, using a notion of adjacency, in which $\Xi$ and $\Xi^{\prime}$ are adjacent if either $\Xi \subset \Xi^{\prime}$ or $\Xi^{\prime} \subset \Xi$. Consider now the identification of the buildings given by the central isogeny $\mathrm{SU}(T) \rightarrow \mathrm{SO}\left(L_{\mathbb{Q}}^{\Phi}\right)$. We can see by looking at the local Dynkin diagrams that Hermitian vertex lattices $\Xi$ of type 0 and 4 are sent to vertex lattices $\Lambda$ of type 6 , and Hermitian vertex lattices $\Xi$ of type 2 are sent to vertex lattices $\Lambda$ of type 2 . Note that $\operatorname{SO}\left(L_{\mathbb{Q}}^{\Phi}\right)$ acts transitively on the set of vertex lattices of type 6 , but the map $\operatorname{SU}(T) \rightarrow \operatorname{SO}\left(L_{\mathbb{Q}}^{\Phi}\right)$ is not surjective on $\mathbb{Q}_{p}$-points: its image is the kernel of the spinor norm.

Consider the set $\mathcal{S}$ which is defined as the disjoint union of the set of Hermitian vertex lattices $\Xi$ with the set of all pairs $\left\{\Xi, \Xi^{\prime}\right\}$ consisting of adjacent Hermitian vertex lattices of types 0 and 4 . Note that there is a natural bijection between the set $\mathcal{S}$ and the set of all vertex lattices $\Lambda$. Hermitian vertex lattices of type 0 and 4 in $\mathcal{S}$ correspond to vertex lattices of type 6, Hermitian vertex lattices of type 2 in $\mathcal{S}$ correspond to vertex lattices of type 2 , and finally the pairs $\left\{\Xi, \Xi^{\prime}\right\}$ correspond to vertex lattices of type 4 .

We define a partial order on $\mathcal{S}$ as follows: For two Hermitian vertex lattices we define $\Xi<\Xi^{\prime}$ if either

(1) $\Xi$ is of type $2, \Xi^{\prime}$ is of type 0 , and $\Xi \subset \Xi^{\prime}$,

(2) $\Xi$ is type $2, \Xi^{\prime}$ is of type 4 , and $\Xi^{\prime} \subset \Xi$ 
(so Hermitian vertex lattices of type 0 and 4 are not comparable). Two pairs $\left\{\Xi_{1}, \Xi_{1}^{\prime}\right\}$ and $\left\{\Xi_{2}, \Xi_{2}^{\prime}\right\}$ in $\mathcal{S}$ are not compared. If $\Xi$ is a Hermitian vertex lattice then $\Xi<\left\{\Xi_{1}, \Xi_{2}\right\}$ if $\Xi, \Xi_{1}$, and $\Xi_{2}$ form a simplex in the building of $J^{\text {der }}$ (which requires that $\Xi$ have type 2). Finally, $\left\{\Xi_{1}, \Xi_{2}\right\}<\Xi$ if $\Xi \in\left\{\Xi_{1}, \Xi_{2}\right\}$. Under the bijection between $\mathcal{S}$ and the set of vertex lattices, this partial order corresponds to inclusion of vertex lattices. Define an adjacency relation in $\mathcal{S}$ by $x \sim \mathcal{S} y$ if either $x<y$ or $y<x$. We also define a dimension function $d: \mathcal{S} \rightarrow\{0,1,2\}$ by $d(x)=0$ if $x$ is a Hermitian vertex lattice of type $2, d(x)=2$ if $x$ is a Hermitian vertex lattice of type 0 or 4 , and $d(x)=1$ if $x$ is a pair $\left\{\Xi, \Xi^{\prime}\right\}$.

The following theorems are simply restatements in this new language of some results of the previous subsection:

Theorem 3.13. Writing the reduced $k$-scheme as a union

$$
\mathscr{M}_{\mathrm{red}}=\biguplus_{\ell \in \mathbb{Z}} \mathscr{M}_{\text {red }}^{(\ell)}
$$

gives the decomposition of $\mathscr{M}_{\mathrm{red}}$ into its connected components $\mathscr{M}_{\mathrm{red}}^{(\ell)}$. These connected components are all isomorphic and are of pure dimension 2.

(1) There is a stratification of $\mathscr{M}_{\text {red }}^{(0)}$ by locally closed smooth subschemes given by

$$
\mathscr{M}_{\mathrm{red}}^{(0)}=\biguplus_{x \in \mathcal{S}} \mathscr{M}_{x}^{\circ}
$$

Each stratum $\mathscr{M}_{x}^{\circ}$ is isomorphic to $\mathscr{N}_{\Lambda}^{+\circ}$, where $\Lambda$ is the vertex lattice that corresponds to $x$, and is therefore isomorphic to a Deligne-Lusztig variety of dimension $d(x)$. The closure $\mathscr{M}_{x}$ of any $\mathscr{M}_{x}^{\circ}$ in $\mathscr{M}_{\mathrm{red}}^{(0)}$ is

$$
\mathscr{M}_{x}=\biguplus_{y \leq x} \mathscr{M}_{y}^{\circ}
$$

(2) We have $\mathscr{M}_{y} \subset \mathscr{M}_{x}$ if and only if $y \leq x$. In particular, the irreducible components of $\mathscr{M}_{\text {red }}^{(0)}$ are precisely the closed subschemes $\mathscr{M}_{\Xi}$ for $\Xi \in \mathcal{S}$ a Hermitian vertex lattice of type 0 or 4.

(3) The schemes $\mathscr{M}_{x}$ are as follows:

(a) If $d(x)=0$, then $\mathscr{M}_{x}$ is a single point.

(b) If $d(x)=1$, then $\mathscr{M}_{x}$ is isomorphic to $\mathbb{P}^{1}$.

(c) If $d(x)=2$, then $\mathscr{M}_{x}$ is isomorphic to the Fermat hypersurface

$$
x_{0}^{p+1}+x_{1}^{p+1}+x_{2}^{p+1}+x_{3}^{p+1}=0 .
$$

Theorem 3.14. The irreducible components of $\mathscr{M}_{\mathrm{red}}^{(0)}$ are parametrized by vertices of type 0 and 4 in the Bruhat-Tits building of $J^{\mathrm{der}}$. Two irreducible components $\mathscr{M}_{\Xi}$ and $\mathscr{M}_{\Xi^{\prime}}$ intersect if and only if $\Xi$ and $\Xi^{\prime}$ are either adjacent, or are adjacent 
to a common element of $\mathcal{S}$. If they are adjacent then one is type 0 , the other of type 4 , and they intersect along $a \mathbb{P}^{1}$. If they are not adjacent but have a common adjacent point $y \in \mathcal{S}$, then $y$ is a Hermitian vertex lattice of type 2 , and $\mathscr{M}_{\Xi} \cap \mathscr{M}_{\Xi^{\prime}}=\mathscr{M}_{y}$ is a single point.

\section{Applications to Shimura varieties}

We now use our explicit description of the Rapoport-Zink space $\mathscr{N}=p^{\mathbb{Z}} \backslash \mathscr{M}$ to describe the supersingular locus of a GU(2,2)-Shimura variety. With the results of Section 3.5 in hand, this is exactly as in the $\operatorname{GU}(n-1,1)$ cases studied in [Rapoport et al. 2014; Vollaard and Wedhorn 2011]. Accordingly, our discussion will be brief.

4.1. The Shimura variety. Let $E \subset \mathbb{C}$ be a quadratic imaginary field, fix a prime $p>2$ inert in $E$, and let $\mathcal{O} \subset E$ be the integral closure of $\mathbb{Z}_{(p)}$ in $E$. Let $V$ be a free $\mathcal{O}$-module of rank 4 endowed with a perfect $\mathcal{O}$-valued Hermitian form $\langle\cdot, \cdot\rangle$ of signature $(2,2)$, and denote by $G=\mathrm{GU}(V)$ the group of unitary similitudes of $V$. It is a reductive group over $\mathbb{Z}_{(p)}$. Fix a compact open subgroup $U^{p} \subset G\left(\mathbb{A}_{f}^{p}\right)$, and define $U_{p}=G\left(\mathbb{Z}_{p}\right)$ and $U=U_{p} U^{p} \subset G\left(\mathbb{A}_{f}\right)$.

The Grassmannian $\mathcal{D}$ of negative-definite planes in $V \otimes_{\mathcal{O}} \mathbb{C}$ is a smooth complex manifold of dimension 4, with an action of $G(\mathbb{R})$. Define

$$
M_{U}(\mathbb{C})=G(\mathbb{Q}) \backslash\left(\mathcal{D} \times G\left(\mathbb{A}_{f}\right) / U\right) .
$$

For sufficiently small $U^{p}$, this is a smooth complex manifold parametrizing primeto- $p$ isogeny classes of quadruples $\left(A, \iota, \lambda,\left[\eta^{p}\right]\right)$, in which $A$ is an abelian variety of dimension $4, \iota: \mathcal{O} \rightarrow \operatorname{End}(A)_{(p)}$ is a ring homomorphism such that

$$
\operatorname{det}(T-\iota(\alpha) ; \operatorname{Lie}(A))=(T-\alpha)^{2}(T-\bar{\alpha})^{2}
$$

for all $\alpha \in \mathcal{O}, \lambda \in \operatorname{Hom}\left(A, A^{\vee}\right)_{(p)}$ is a prime-to-p-quasi-polarization satisfying

$$
\lambda \circ \iota(\bar{\alpha})=\iota(\alpha)^{\vee} \circ \lambda
$$

for all $\alpha \in \mathcal{O}$, and $\left[\eta^{p}\right]$ is the $U^{p}$-orbit of an $\mathcal{O} \otimes \mathbb{A}_{f}^{p}$-linear isomorphism

$$
\eta^{p}: \widehat{\mathrm{Ta}}^{p}(A) \otimes \mathbb{A}_{f}^{p} \rightarrow V \otimes \mathbb{A}_{f}^{p}
$$

respecting the Hermitian forms up to scaling by $\left(\mathbb{A}_{f}^{p}\right)^{\times}$(the Hermitian form on the source is determined by $\lambda$, as in (2-3)). A prime-to-p-isogeny between two such pairs $\left(A, \iota, \lambda,\left[\eta^{p}\right]\right)$ and $\left(A^{\prime}, \iota^{\prime}, \lambda^{\prime},\left[\eta^{p^{\prime}}\right]\right)$ is an $\mathcal{O}$-linear quasi-isogeny in $\operatorname{Hom}\left(A, A^{\prime}\right)_{(p)}$ of degree prime to $p$ that respects the level structures, and such that $\lambda^{\prime}$ pulls back to a $\mathbb{Z}_{(p)}^{\times}$-multiple of $\lambda$.

The parametrization is similar to the constructions found in [Kudla and Rapoport 2009], and can be described as follows. For each triple $(A, i, \lambda,[\eta])$ above, the 
existence of $\eta^{p}$ implies that $H_{1}(A, \mathbb{Q})$ and $V \otimes \mathbb{Q}$ are isomorphic, as Hermitian spaces, locally at all places $v \nmid p$. But this implies that they are also isomorphic at $p$, and hence there is a global isomorphism

$$
\beta: H_{1}(A, \mathbb{Q}) \rightarrow V \otimes \mathbb{Q} .
$$

As $\operatorname{Ta}_{p}(A) \otimes \mathbb{Q}_{p} \cong V \otimes \mathbb{Q}_{p}$, a result of Jacobowitz, stated in [Kudla and Rapoport 2009, Proposition 2.14], shows that there is a unique $U_{p}$-orbit of isomorphisms $\operatorname{Ta}_{p}(A) \cong V \otimes \mathbb{Z}_{p}$ compatible with the $\mathcal{O}$-actions and Hermitian forms. Thus there is a unique way to extend $\eta^{p}$ to a $U$-orbit of isomorphisms

$$
\eta: \hat{\operatorname{Ta}}(A) \otimes \mathbb{A}_{f} \cong V \otimes \mathbb{A}_{f}
$$

compatible with the $\mathcal{O}$-actions and the symplectic forms, and identifying $\operatorname{Ta}_{p}(A)$ with $V \otimes \mathbb{Z}_{p}$. The composition

$$
V \otimes \mathbb{A}_{f} \stackrel{\eta^{-1}}{\longrightarrow} H_{1}\left(A, \mathbb{A}_{f}\right) \stackrel{\beta}{\rightarrow} V \otimes \mathbb{A}_{f}
$$

defines an element $g \in G\left(\mathbb{A}_{f}\right) / U$, and the Hodge structure on $V \otimes \mathbb{R}$ induced by the isomorphism $\beta$ corresponds to a point of $\mathcal{D}$, as in [Kudla and Rapoport 2009, Section 3].

4.2. The uniformization theorem. Let $k$ be an algebraic closure of the field of $p$ elements.

Extending the moduli problem of the previous subsection to $\mathbb{Z}_{(p)}$-schemes in the obvious way yields a scheme $M_{U}$, smooth of relative dimension 4 over $\mathbb{Z}_{(p)}$. Denote by $M_{U}^{\text {ss }}$ the reduced supersingular locus of the geometric special fiber $M_{U} \times_{\mathbb{Z}_{(p)}} k$. A choice of geometric point $(\boldsymbol{A}, \boldsymbol{\iota}, \boldsymbol{\lambda},[\boldsymbol{\eta}]) \in M_{U}^{\text {ss }}(k)$ determines a base point $(\boldsymbol{G}, \boldsymbol{\iota}, \boldsymbol{\lambda})$ with $\boldsymbol{G}=\boldsymbol{A}\left[p^{\infty}\right]$, and so defines a Rapoport-Zink space $\mathscr{M}$ as in Section 2.1, endowed with an action of the subgroup $J \subset \operatorname{End}(\boldsymbol{G})_{\mathbb{Q}}^{\times}$. Denote by $I(\mathbb{Q}) \subset \operatorname{End}(A)_{\mathbb{Q}}^{\times}$ the subgroup of $\mathcal{O}$-linear quasi-automorphisms that preserve the $\mathbb{Q}^{\times}$-span of $\lambda$. It is the group of $\mathbb{Q}$-points of an algebraic group $I$ over $\mathbb{Q}$ satisfying $I\left(\mathbb{Q}_{p}\right) \cong J$, and the orbit $[\eta]$ determines a right $U^{p}$-orbit of isomorphisms $I\left(\mathbb{A}_{f}^{p}\right) \cong G\left(\mathbb{A}_{f}^{p}\right)$. In particular, $I(\mathbb{Q})$ acts on both $\mathscr{M}$ and on $G\left(\mathbb{A}_{f}^{p}\right) / U^{p}$.

Theorem 4.1 (Rapoport-Zink). There is an isomorphism of $k$-schemes

$$
M_{U}^{\mathrm{ss}} \cong I(\mathbb{Q}) \backslash\left(\mathscr{M}_{\mathrm{red}} \times G\left(\mathbb{A}_{f}^{p}\right) / U^{p}\right) .
$$

As in [Vollaard 2010, Corollary 6.2], combining the above uniformization theorem with the results of Section 3.5 yields the following corollary:

Corollary 4.2. The $k$-scheme $M_{U}^{\text {ss }}$ has pure dimension 2 . For $U^{p}$ sufficiently small, all irreducible components of $M_{U}^{\text {ss }}$ are isomorphic to the Fermat hypersurface

$$
x_{0}^{p+1}+x_{1}^{p+1}+x_{2}^{p+1}+x_{3}^{p+1}=0,
$$


and any two irreducible components either intersect trivially, intersect at a single point, or their intersection is isomorphic to $\mathbb{P}^{1}$. Here "intersection" is understood to mean the reduced scheme underlying the scheme-theoretic intersection.

\section{Acknowledgements}

The authors would like to thank M. Rapoport for many useful suggestions, and $\mathrm{X}$. He for communicating his joint results with U. Görtz.

\section{References}

[Bass 1974] H. Bass, "Clifford algebras and spinor norms over a commutative ring", Amer. J. Math. 96 (1974), 156-206. MR 50 \#13092 Zbl 0344.15017

[Bonnafé and Rouquier 2006] C. Bonnafé and R. Rouquier, "On the irreducibility of Deligne-Lusztig varieties", C. R. Math. Acad. Sci. Paris 343:1 (2006), 37-39. MR 2007a:14055 Zbl 1099.14040

[Bruhat and Tits 1984] F. Bruhat and J. Tits, "Groupes réductifs sur un corps local, II: Schémas en groupes. Existence d'une donnée radicielle valuée”, Inst. Hautes Études Sci. Publ. Math. 60 (1984), 197-376. MR 86c:20042 Zbl 0597.14041

[Bültel 2012] O. Bültel, "On the supersingular loci of quaternionic Siegel space”, preprint, 2012. arXiv 1209.3548

[Deligne and Lusztig 1976] P. Deligne and G. Lusztig, "Representations of reductive groups over finite fields", Ann. of Math. (2) 103:1 (1976), 103-161. MR 52 \#14076 Zbl 0336.20029

[Freitag and Hermann 2000] E. Freitag and C. F. Hermann, "Some modular varieties of low dimension”, Adv. Math. 152:2 (2000), 203-287. MR 2001j:11023 Zbl 0974.11028

[Garrett 1997] P. Garrett, Buildings and classical groups, Chapman \& Hall, London, 1997. MR 98k: 20081 Zbl 0933.20019

[Göertz and He 2013] U. Göertz and X. He, "Basic loci in Shimura varieties of Coxeter type", preprint, 2013. arXiv 1311.6263

[Kim 2013] W. Kim, "Rapoport-Zink spaces of Hodge type”, preprint, 2013. arXiv 1308.5537

[Kisin 2010] M. Kisin, "Integral models for Shimura varieties of abelian type", J. Amer. Math. Soc. 23:4 (2010), 967-1012. MR 2011j:11109 Zbl 1280.11033

[Knus et al. 1998] M.-A. Knus, A. Merkurjev, M. Rost, and J.-P. Tignol, The book of involutions, American Mathematical Society Colloquium Publications 44, American Mathematical Society, Providence, RI, 1998. MR 2000a:16031 Zbl 0955.16001

[Kudla and Rapoport 1999] S. S. Kudla and M. Rapoport, "Arithmetic Hirzebruch-Zagier cycles", J. Reine Angew. Math. 515 (1999), 155-244. MR 2002e:11076a Zbl 1048.11048

[Kudla and Rapoport 2000] S. S. Kudla and M. Rapoport, "Cycles on Siegel threefolds and derivatives of Eisenstein series", Ann. Sci. École Norm. Sup. (4) 33:5 (2000), 695-756. MR 2002e:11076b Zbl 1045.11044

[Kudla and Rapoport 2009] S. Kudla and M. Rapoport, "Special cycles on unitary Shimura varieties II: global theory", preprint, 2009. arXiv 0912.3758

[Kudla and Rapoport 2011] S. Kudla and M. Rapoport, "Special cycles on unitary Shimura varieties, I: Unramified local theory”, Invent. Math. 184:3 (2011), 629-682. MR 2012k:14033 Zbl 1229.14020

[Lang 2002] S. Lang, Algebra, 3rd ed., Graduate Texts in Mathematics 211, Springer, New York, 2002. MR 2003e:00003 Zbl 0984.00001 
[Madapusi Pera 2012] K. Madapusi Pera, "Integral canonical models for Spin Shimura varieties", preprint, 2012. arXiv 1212.1243

[Rapoport and Zink 1996] M. Rapoport and T. Zink, Period spaces for p-divisible groups, Annals of Mathematics Studies 141, Princeton University Press, 1996. MR 97f:14023 Zbl 0873.14039

[Rapoport et al. 2014] M. Rapoport, U. Terstiege, and S. Wilson, "The supersingular locus of the Shimura variety for GU(1,n-1) over a ramified prime”, Math. Z. 276:3-4 (2014), 1165-1188. MR 3175176 Zbl 06304108

[Shimura 2010] G. Shimura, Arithmetic of quadratic forms, Springer Monographs in Mathematics, Springer, New York, 2010. MR 2011m:11003 Zbl 1202.11026

[Springer 1998] T. A. Springer, Linear algebraic groups, 2nd ed., Progress in Mathematics 9, Birkhäuser, Boston, 1998. MR 99h:20075 Zbl 0927.20024

[Tits 1979] J. Tits, "Reductive groups over local fields", pp. 29-69 in Automorphic forms, representations and L-functions, Part 1 (Corvallis, OR, 1977), edited by A. Borel and W. Casselman, Proc. Sympos. Pure Math., XXXIII 33, Amer. Math. Soc., Providence, R.I., 1979. MR 80h:20064 Zbl 0415.20035

[Vasiu 1999] A. Vasiu, "Integral canonical models of Shimura varieties of preabelian type", Asian J. Math. 3:2 (1999), 401-518. MR 2002b:11087 Zbl 1002.11052

[Vollaard 2010] I. Vollaard, "The supersingular locus of the Shimura variety for GU(1,s)", Canad. J. Math. 62:3 (2010), 668-720. MR 2011j:14059 Zbl 1205.14028

[Vollaard and Wedhorn 2011] I. Vollaard and T. Wedhorn, "The supersingular locus of the Shimura variety of GU(1, $n-1)$ II”, Invent. Math. 184:3 (2011), 591-627. MR 2012j:14035 Zbl 1227.14027

[Zink 2001] T. Zink, "Windows for displays of p-divisible groups", pp. 491-518 in Moduli of abelian varieties (Texel, 1999), edited by C. Faber et al., Progr. Math. 195, Birkhäuser, Basel, 2001. MR 2002c:14073 Zbl 1099.14036

Communicated by Barry Mazur

Received 2013-10-16 Revised 2014-02-17 Accepted 2014-05-23

howardbe@bc.edu

Department of Mathematics, Boston College, Chestnut Hill, MA 02467-3806, United States

pappas@math.msu.edu

Department of Mathematics, Michigan State University, East Lansing, MI 48824-1027, United States 


\section{Algebra \& Number Theory}

msp.org/ant

\section{EDITORS}

MANAGING EDITOR

Bjorn Poonen

Massachusetts Institute of Technology

Cambridge, USA

\author{
EDITORIAL BOARD CHAIR \\ David Eisenbud \\ University of California \\ Berkeley, USA
}

BOARD OF EDITORS

Georgia Benkart

Dave Benson

Richard E. Borcherds

John H. Coates

J-L. Colliot-Thélène

Brian D. Conrad

Hélène Esnault

Hubert Flenner

Edward Frenkel

Andrew Granville

Joseph Gubeladze

Roger Heath-Brown

Craig Huneke

Yujiro Kawamata

János Kollár

Yuri Manin

Barry Mazur

Philippe Michel

Susan Montgomery
University of Wisconsin, Madison, USA

University of Aberdeen, Scotland

University of California, Berkeley, USA

University of Cambridge, UK

CNRS, Université Paris-Sud, France

University of Michigan, USA

Freie Universität Berlin, Germany

Ruhr-Universität, Germany

University of California, Berkeley, USA

Université de Montréal, Canada

San Francisco State University, USA

Oxford University, UK

University of Virginia, USA

University of Tokyo, Japan

Princeton University, USA

Northwestern University, USA

Harvard University, USA

École Polytechnique Fédérale de Lausanne

University of Southern California, USA
Shigefumi Mori

Raman Parimala

Jonathan Pila

Anand Pillay

Victor Reiner

Peter Sarnak

Joseph H. Silverman

Michael Singer

Vasudevan Srinivas

J. Toby Stafford

Bernd Sturmfels

Richard Taylor

Ravi Vakil

Michel van den Bergh

Marie-France Vignéras

Kei-Ichi Watanabe

Efim Zelmanov

Shou-Wu Zhang
RIMS, Kyoto University, Japan

Emory University, USA

University of Oxford, UK

University of Notre Dame, USA

University of Minnesota, USA

Princeton University, USA

Brown University, USA

North Carolina State University, USA

Tata Inst. of Fund. Research, India

University of Michigan, USA

University of California, Berkeley, USA

Harvard University, USA

Stanford University, USA

Hasselt University, Belgium

Université Paris VII, France

Nihon University, Japan

University of California, San Diego, USA

Princeton University, USA

\section{PRODUCTION}

production@msp.org

Silvio Levy, Scientific Editor

See inside back cover or msp.org/ant for submission instructions.

The subscription price for 2014 is US $\$ 225 /$ year for the electronic version, and $\$ 400 /$ year ( $\$ 55$, if shipping outside the US) for print and electronic. Subscriptions, requests for back issues and changes of subscribers address should be sent to MSP.

Algebra \& Number Theory (ISSN 1944-7833 electronic, 1937-0652 printed) at Mathematical Sciences Publishers, 798 Evans Hall \#3840, c/o University of California, Berkeley, CA 94720-3840 is published continuously online. Periodical rate postage paid at Berkeley, CA 94704, and additional mailing offices.

ANT peer review and production are managed by EditFLOW ${ }^{\circledR}$ from MSP.

\section{PUBLISHED BY}

- mathematical sciences publishers

nonprofit scientific publishing

http://msp.org/

(C) 2014 Mathematical Sciences Publishers 


\section{Algebra \& Number Theory}

Volume $8 \quad$ No. $7 \quad 2014$

Double Dirichlet series and quantum unique ergodicity of weight one-half Eisenstein series

Yiannis N. Petridis, Nicole RaUlF and Morten S. Risager

Monodromy and local-global compatibility for $l=p$

ANA CARAIANI

Finite generation of the cohomology of some skew group algebras

VAN C. NGUYEN and SARAH WITHERSPOON

On the supersingular locus of the $G U(2,2)$ Shimura variety

BENJAMIN HOWARD and GEORgIOS PAPPAS

Poincaré-Birkhoff-Witt deformations of smash product algebras from Hopf actions on

1701

Koszul algebras

Chelsea Walton and Sarah Witherspoon

Highly biased prime number races

1733

DANIEL FIORILLI

Bounded gaps between primes with a given primitive root

1769

PAUL POLlACK 